TOKYO J. MATH.

VOL. 36, No. 2, 2013

\title{
The Lefschetz Elements of Coinvariant Algebras of Binary Polyhedral Groups
}

\author{
Sachiko YOSHIDA \\ Nara Women's University \\ (Communicated by J. Murakami)
}

\begin{abstract}
In this paper, we give a characterization of the Lefschetz elements in a coinvariant algebra of an arbitrary complex reflection group which contains a binary polyhedral group as a subgroup of index 2 . In addition, we examine the relation between the set of the non-strong Lefschetz elements and polyhedra.
\end{abstract}

\section{Introduction}

The notion of the Lefschetz properties comes from the hard Lefschetz theorem in complex geometry. The hard Lefschetz theorem holds for the flag varieties in particular. The cohomology ring of the flag variety is isomorphic to the coinvariant algebra of the corresponding Weyl group $W$, which is the regular representation of $W$.

In general the coinvariant algebras of finite groups are not always isomorphic to the cohomology rings of any manifolds. Indeed the coinvariant algebras of non-crystallographic finite Coxeter groups are not isomorphic to the cohomology rings of any varieties, but have the Lefschetz properties ([6], [9], [10], [13]). See [2], [3], [7], [13], [14], for the study of Lefschetz properties for wider classes of graded Artinian algebras.

In this paper, we give characterizations of the strong Lefschetz elements for an arbitrary complex reflection group $G$ which contains a finite subgroup of $S L(2, \mathbf{C})$ as a subgroup of index 2. Furthermore we also characterize the weak Lefschetz elements for the complex reflection groups, a dihedral group and $G(2 n, n, 2)$, which contain a cyclic group and a binary dihedral group respectively. The set of the strong Lefschetz elements is the complement of the union of the zero locus of $G$-semi-invariant homogeneous polynomials $g_{i}$. The set of polynomials $g_{i}$ contains $G$-invariants whose zeros correspond to the vertices, the centers of the edges and of the faces of the corresponding regular polyhedrons.

Let $p: S^{2} \longrightarrow \mathbf{C} \cup\{\infty\}=\mathbf{P}^{1}$ be the stereographic projection. If we put $t\left(g_{i}\right)=$ $\left\{(a: b) \in \mathbf{P}^{1} \mid g_{i}(a, b)=0\right\}$, then the inverse image $p^{-1}\left(t\left(g_{i}\right)\right)$ in $S^{2}$ consists of the points corresponding to the vertices, the centers of the edges, the centers of the faces of the regular polyhedrons, and generic points of $S^{2}$.

Received April 23, 2012; revised October 2, 2012 
This paper is organized as follows. In section 2, we collect some basic facts on binary polyhedral groups and complex reflection groups containing binary polyhedral groups. In section 3, we introduce the definition of the coinvariant algebras and the Lefschetz properties. In the remaining sections, we determine the strong Lefschetz elements for an arbitrary complex reflection group containing a finite subgroup of $S L(2, \mathbf{C})$.

ACKNOWLEDGMENTS. The author would like to express her many thanks to Professor J. Matsuzawa for all his encouragement and to Professor T. Maeno for generous support. The author would also like to express her gratitude to Professor J. Watanabe and Professor A. Wachi for many helpful suggestions. Finally the author is very grateful to Professor T. Morimoto for giving her many valuable suggestions.

\section{Binary polyhedral groups and complex reflection groups}

2.1. Binary polyhedral groups. In this section, we explain the basic results on binary polyhedral groups, which show that every finite subgroup of $\operatorname{SL}(2, \mathbf{C})$ is isomorphic to a cyclic group or a binary polyhedral group.

It is known that the regular polyhedra are classified into 5 types : regular tetrahedron, cube, regular octahedron, regular dodecahedron, regular icosahedron. When the barycenter of a regular polyhedron is put on the origin of three-dimensional Euclidean space $\mathbf{R}^{\mathbf{3}}$, the vertices of a regular polyhedron are on the sphere $S^{2}$.

For a regular polyhedron $\Delta$, let $\Gamma_{\Delta}$ be the subgroup of rotation group $S O$ (3) consisting of the rotations that transform $\Delta$ into itself.

$$
\Gamma_{\Delta}=\{g \in S O(3) \mid g(\Delta)=\Delta\}
$$

Besides the groups of the regular polyhedra, there are two more infinite series of finite subgroups of $S O(3)$ : cyclic groups and dihedral groups.

Any convex solid has a dual namely the convex hull of the barycenters of its faces. By this convex duality, the octahedron and cube, resp. the icosahedron and dodecahedron, give rise to the same group.

Now $S^{2}$ is considered as the Riemannian sphere, i.e. as the complex number plane $\mathbf{C}$ of the complex variable $z$ compactified by one point $\infty$. We identify the sphere $S^{2}$ with $\mathbf{C} \cup\{\infty\}$ by the stereographic projection.

After identifying $\mathbf{C} \cup\{\infty\}$ with the complex projective line $\mathbf{P}^{1}(\mathbf{C})$, any rotation $g \in$ $S O$ (3) acting on $S^{2}$ corresponds to a fractional linear transformation $z \mapsto \frac{a z+b}{c z+d}$ of $\mathbf{C} \cup$ $\{\infty\}$. By this correspondence, $S O(3)$ is considered as the subgroup of complex projective transformation group

$$
P G L(2, \mathbf{C})=P S L(2, \mathbf{C})=S L(2, \mathbf{C}) /\langle \pm 1\rangle
$$

which is the automorphism group of $\mathbf{P}^{1}(\mathbf{C})$. 
The inverse image of $S O(3)$ under the projection

$$
\pi: S L(2, \mathbf{C}) \longrightarrow \operatorname{PSL}(2, \mathbf{C})
$$

is the special unitary group :

$$
S U(2)=\pi^{-1}(S O(3))
$$

Definition 1. A subgroup of $S L(2, \mathbf{C})$ which is conjugate to the inverse image $\pi^{-1}(\Gamma)$ of the finite subgroup $\Gamma$ of $S O(3)$ is called a binary subgroup corresponding to $\Gamma$. We denote this by $G$.

When $\Gamma$ is a polyhedral group, $G$ is called a binary polyhedral group.

Since the kernel of $\pi$ is $\{I,-I\}$ ( $I$ is the unit matrix), we have

$$
|G|=2|\Gamma| \text {. }
$$

THEOREM 1 (See e.g. [12]). There are the following conjugacy classes of finite subgroups of $\operatorname{SL}(2, \mathbf{C})$.

1. cyclic groups (order $n, n \in \mathbf{N}$ )

2. binary dihedral groups (order $4 n, n \in \mathbf{N}$ )

3. binary tetrahedral groups (order 24)

4. binary octahedral groups (order 48)

5. binary icosahedral groups (order 120)

In this paper, the polyhedral group is the general term for dihedral groups and the groups of the regular polyhedra.

EXAMPLE 1. (1) cyclic group $C_{n}(\operatorname{order} n)$

$$
C_{n}=\left\{\left(\begin{array}{cc}
\theta^{h} & 0 \\
0 & \theta^{-h}
\end{array}\right) \mid(0 \leq h<n)\right\}, \quad \theta=e^{\frac{2 \pi i}{n}}, \quad(n \geq 2)
$$

(2) binary dihedral group $\widetilde{D}_{n}($ order $4 n)$

$$
\widetilde{D}_{n}=\left\langle\left(\begin{array}{cc}
\theta & 0 \\
0 & \theta^{-1}
\end{array}\right),\left(\begin{array}{cc}
0 & 1 \\
-1 & 0
\end{array}\right)\right\rangle, \quad \theta=e^{\frac{\pi i}{n}}, \quad(n \geq 3)
$$

2.2. Complex reflection groups including binary polyhedral groups. A reflection on complex vector space is a congruent transformation of finite period that leaves invariant every point of a hyperplane, and it is characterized by the property that all but one of the characteristic roots of the matrix of transformation are equal to unity.

Let $G$ a finite subgroup of $S L(2, \mathbf{C})$. There exists a complex reflection group $\tilde{G}$ of $G L(2, \mathbf{C})$ containing $G$ with $[\tilde{G}: G]=2$ ([11]).

We list all such pairs $G$ and $\tilde{G}$.

We use the notation in [11]. 


\begin{tabular}{cccc}
\hline$G$ & order & $\tilde{G}$ & order \\
\hline cyclic group & $n$ & dihedral group & $2 n$ \\
binary dihedral group & $4 n$ & $G(2 n, n, 2)$ & $8 n$ \\
binary tetrahedral group & 24 & $G_{12}$ & 48 \\
binary octahedral group & 48 & $G_{13}$ & 96 \\
binary icosahedral group & 120 & $G_{22}$ & 240 \\
\hline
\end{tabular}

EXAMPLE 2. (1) dihedral group $D_{n}$

$$
C_{n} \subset D_{n}=\left\langle\left(\begin{array}{cc}
\theta & 0 \\
0 & \theta^{-1}
\end{array}\right),\left(\begin{array}{ll}
0 & 1 \\
1 & 0
\end{array}\right)\right\rangle, \quad \theta=e^{\frac{2 \pi i}{n}}, \quad(n \geq 3)
$$

(2) $G(2 n, n, 2)$

$$
\widetilde{D_{n}} \subset G(2 n, n, 2)=\left\langle\left(\begin{array}{ll}
0 & 1 \\
1 & 0
\end{array}\right),\left(\begin{array}{cc}
-1 & 0 \\
0 & 1
\end{array}\right),\left(\begin{array}{cc}
\theta & 0 \\
0 & \theta^{-1}
\end{array}\right)\right\rangle, \quad \theta=e^{\frac{\pi i}{n}}, \quad(0 \leq k \leq 2 n-1)
$$

\section{Coinvariant algebra}

In this section we define the coinvariant algebra for a finite group and introduce the notion of the strong Lefschetz property and the weak Lefschetz property for graded Artinian algebras over the field $\mathbf{C}$.

3.1. Invariant algebra. Let $V$ denote a complex vector space of dimension $n$ and $V^{*}$ its dual vector space. If $\operatorname{Sym}\left(V^{*}\right)$ denotes the symmetric algebra over $V^{*}=\operatorname{Hom}_{\mathbf{C}}(V, \mathbf{C})$, the polynomial algebra on $V$ is

$$
S(V)=\operatorname{Sym}\left(V^{*}\right)=\bigoplus_{j=0}^{\infty} S_{j}(V),
$$

where $S_{j}(V)$ is the space of homogeneous polynomials of degree $j$.

If we choose a basis $\left\{e_{1}, e_{2}, \ldots, e_{n}\right\}$ for $V$ and let $\left\{x_{1}, x_{2}, \ldots, x_{n}\right\}$ denote the dual basis of $V^{*}$, i.e. $x_{i}\left(e_{j}\right)=\delta_{i j}$, then $S(V)$ can be identified with the polynomial algebra $\mathbf{C}\left[x_{1}, x_{2}, \ldots, x_{n}\right]$ :

$$
S(V)=\mathbf{C}\left[x_{1}, x_{2}, \ldots, x_{n}\right]=\operatorname{Sym}\left(V^{*}\right)
$$

Let $F$ be a finite group acting on a complex vector space $V$. We define the action of $F$ on $S(V)$ by

$$
g \cdot \phi(z)=\phi\left(g^{-1}(z)\right), \quad g \in F, \quad \phi \in S(V), \quad z \in V
$$


Definition 2. For all $g \in F$, we call $\phi \in S(V)$ an $F$-invariant if

$$
g \cdot \phi=\phi \text {. }
$$

The set of $F$-invariants

$$
S(V)^{F}:=\{\phi \in S(V) \mid g \cdot \phi=\phi\}
$$

forms a subalgebra of $S(V)$ and is called a invariant algebra.

THEOREM 2 (See e.g. [12], [chap. 4]). The following properties of the finite group $F$ are equivalent.

1. F is a finite reflection group.

2. $S(V)$ is a free graded module over $S(V)^{F}$ with a finite basis.

3. $S(V)^{F}$ is generated by $n$ algebraically independent homogeneous elements.

\subsection{Coinvariant algebra and the Lefschetz properties}

DEFINITION 3. If $F$ acts on $V$, let $I_{F}$ denote the homogeneous ideal in $S(V)$ generated by $S(V)_{+}^{F}=\bigoplus_{i>0} S_{i}(V)^{F}$.

Then we call the graded quotient algebra

$$
S(V)_{F}=S(V) / I_{F}=\bigoplus_{i=0}^{\infty} S_{i}(V)_{F}
$$

the coinvariant algebra.

For a graded vector space $R=\bigoplus_{i \geq 0} R_{i}$, over $\mathbf{C}$, let

$$
P_{R}(t)=\sum_{i \geq 0} \operatorname{dim}_{\mathbf{C}}\left(R_{i}\right) t^{i}
$$

be the Poincaré series of $R$.

By Theorem 2, we have

$$
\begin{array}{r}
P_{S(V)_{F}}(t)=\frac{P_{S(V)}(t)}{P_{S(V)^{F}}(t)}, \\
P_{S(V)}(t)=(1-t)^{-n}, \\
P_{S(V)^{F}}(t)=\prod_{i=1}^{n}\left(1-t^{d_{i}}\right)^{-1},
\end{array}
$$

where $n=\operatorname{dim}(V)$ and $d_{1}, d_{2}, \ldots, d_{n}$ are the degrees of algebraically independent generators of $S(V)^{F}$.

We list the degrees of algebraically independent generators of the complex reflection group $\tilde{G}$ of $G L(2, \mathbf{C})$ containing $G$ ([11]). 
TABLE 1

\begin{tabular}{cc}
\hline$\tilde{G}$ & degree \\
\hline dihedral group & $2, n$ \\
\hline$G(2 n, n, 2)$ & $4,2 n$ \\
\hline$G_{12}$ & 6,8 \\
\hline$G_{13}$ & 8,12 \\
\hline$G_{22}$ & 12,20 \\
\hline
\end{tabular}

Definition 4. Let $A=\bigoplus_{i=0}^{D} A_{i}, A_{D} \neq 0$, be a commutative Artinian graded algebra.

(1) We say that $A$ has the strong Lefschetz property if there exists an element $l \in A_{1}$ such that the map given by multiplying $l^{D-2 i}$

$$
\sigma_{i}: A_{i} \longrightarrow A_{D-i}
$$

is bijective for $i=0, \ldots,\left[\frac{D}{2}\right]$.

We call $l \in A_{1}$ with this property a strong Lefschetz element.

(2) We say that $A$ has the weak Lefschetz property if there exists an element $l \in A_{1}$ such that the map given by multiplying $l$

$$
\tau_{i}: A_{i} \longrightarrow A_{i+1}
$$

is of full rank for $i=0, \ldots, D-1$.

We call $l \in A_{1}$ with this property a weak Lefschetz element.

Let $G$ be a finite subgroup of $S L(2, \mathbf{C})$ acting on a 2-dimensional complex vector space $V$. Let $\tilde{G}$ be a complex reflection group containing $G$ as a subgroup of index 2 . Then for the coinvariant algebras

$$
S(V)_{\tilde{G}}=\bigoplus_{i=0}^{\bar{d}} S_{i}(V)_{\tilde{G}}, \quad S(V)_{G}=\bigoplus_{i=0}^{d} S_{i}(V)_{G},
$$

we have (see e.g. [1])

\section{PROPOSITION 1 .}

1. $\bar{d}=d+1$

2. $I_{\tilde{G}}=I_{G} \oplus \mathbf{C} f, S_{\bar{d}}(V)_{\tilde{G}}=\mathbf{C} f$.

3. The algebra homomorphism $\varphi: S(V)_{\tilde{G}} \rightarrow S(V)_{G}$ induces the isomorphisms $S_{i}(V)_{\tilde{G}} \rightarrow S_{i}(V)_{G}$ for $i \leq d$ as vector spaces.

This proposition shows that the studies of the Lefschetz elements of $S(V)_{\tilde{G}}$ and $S(V)_{G}$ are much the same. Therefore we investigate the Lefschetz properties for $\tilde{G}$. Since the coin- 
variant algebra $S(V)_{\tilde{G}}$ is the quotient of $\mathbf{C}[x, y], S(V)_{\tilde{G}}$ has the strong Lefschetz property ([2]). In the following sections, we give characterizations of the strong Lefschetz elements for $\tilde{G}$.

For later use, we introduce the following notations.

1. Let $\rho: S(V) \longrightarrow S(V)_{G}$ the homomorphism from $S(V)$ to the coinvariant algebra $S(V)_{G}:=S(V) / I_{G}$ given by $x=\rho(X), y=\rho(Y)(X, Y \in S(V))$.

2. Let

$$
p: S^{2} \longrightarrow \mathbf{C} \cup\{\infty\}=\mathbf{P}^{1}
$$

be the stereographic projection. For a homogeneous polynomial $g_{i} \in \mathbf{C}[x, y]$, put

$$
t\left(g_{i}\right)=\left\{(a: b) \in \mathbf{P}^{1} \mid g_{i}(a, b)=0\right\}
$$

and

$$
B\left(g_{i}\right):=p^{-1}\left(t\left(g_{i}\right)\right)
$$

\section{The Lefschetz elements for dihedral group}

We recall that the dihedral group of order $2 n$ contains a cyclic group of order $n$ as a subgroup of index 2. In this section, we give a characterization of the strong (weak) Lefschetz element of the coinvariant algebras of the dihedral groups.

Let $G$ be a dihedral group :

$$
G=\left\langle\left(\begin{array}{cc}
\theta & 0 \\
0 & \theta^{-1}
\end{array}\right),\left(\begin{array}{ll}
0 & 1 \\
1 & 0
\end{array}\right)\right\rangle, \quad \theta=e^{\frac{2 \pi i}{n}}, \quad n \geq 3 .
$$

The invariant algebra of $G$ is

$$
S(V)^{G}=\mathbf{C}\left[X^{n}+Y^{n}, X Y\right] .
$$

By (3) in 3.2 and Table 1, we have

$$
\begin{aligned}
P_{S(V)_{G}}(t) & =\frac{\left(1-t^{2}\right)\left(1-t^{n}\right)}{(1-t)^{2}} \\
& =1+2 t+2 t^{2}+\cdots+2 t^{n-1}+t^{n} .
\end{aligned}
$$

We choose a basis of $S(V)_{G}$

$$
\left\{1, x, y, x^{2}, y^{2}, \ldots, x^{n-1}, y^{n-1}, x^{n}\right\} .
$$

THEOREM 3. (1) The element $l=a x+b y \in S_{1}(V)_{G}$ is the strong Lefschetz element if and only if $\prod_{i=1}^{3} g_{i}(a, b) \neq 0$, where $g_{i}, 1 \leq i \leq 3$, are the polynomials in the variables $a, b$ given by

$$
g_{1}=a, \quad g_{2}=b, \quad g_{3}=a^{n}-b^{n} .
$$


(See the end of $\$ 3$ for the notation.)

(2) The element $l=a x+b y \in S_{1}(V)_{G}$ is the weak Lefschetz element if and only if $a \neq 0$ and $b \neq 0$.

(3) We have the inverse images of $t\left(g_{i}\right)$ under the stereographic projection as follows (see the end of $\$ 3$ for the notation) :

$$
\begin{gathered}
B\left(g_{1}\right)=\{(0,0,-1)\}, \\
B\left(g_{2}\right)=\{(0,0,1)\}, \\
B\left(g_{3}\right)=\left\{\left(\cos \frac{2 \pi k}{n}, \sin \frac{2 \pi k}{n}, 0\right) \mid 0 \leq k \leq n-1\right\} .
\end{gathered}
$$

We take an $n$-sided regular polygon on XY-plane. Now if we put a vertex of the regular polygon on $(1,0,0)$, the set $B\left(g_{i}\right)$ are given as follows:

(i) $B\left(g_{1}\right) \cup B\left(g_{2}\right)$ is the set of points corresponding to the centers of the faces of the regular polygon.

(ii) $B\left(g_{3}\right)$ is the set of $n$ vertices of the regular polygon.

Proof. (1) Since $I_{G}=\left(X^{n}+Y^{n}, X Y\right)$, we have

$$
x y=0, \quad x^{n}+y^{n}=0 .
$$

By (4.1), the matrix representation of $\sigma_{0}$ and $\sigma_{i}\left(1 \leq i \leq\left[\frac{n}{2}\right]\right)$ with respect to the basis (4.0) are

$$
\left(a^{n}-b^{n}\right), \quad\left(\begin{array}{cc}
a^{n-2 i} & 0 \\
0 & b^{n-2 i}
\end{array}\right)
$$

respectively. Thus we get (1).

(2) By (4.1), the matrix representation of $\tau_{0}, \tau_{i}(1 \leq i \leq n-2), \tau_{n-1}$ with respect to the basis (4.0) are

$$
\left(\begin{array}{l}
a \\
b
\end{array}\right), \quad\left(\begin{array}{ll}
a & 0 \\
0 & b
\end{array}\right), \quad(a-b)
$$

respectively. Thus we get (2).

(3) For a homogeneous polynomial $g_{i}$, the points $(a: b) \in t\left(g_{i}\right)$ are as follows:

$$
\begin{gathered}
t\left(g_{1}\right)=\{(0: 1)\}, \\
t\left(g_{2}\right)=\{(1: 0)\}, \\
t\left(g_{3}\right)=\left\{\left(\theta^{k}: 1\right) \mid k=0, \ldots, n-1\right\}, \theta=e^{\frac{2 \pi \sqrt{-1}}{n}} .
\end{gathered}
$$


We identify the sphere $S^{2}$ with $\mathbf{C} \cup\{\infty\}$ by the stereographic projection $p$ :

$$
\xi=\frac{2 \alpha}{1+\alpha^{2}+\beta^{2}}, \quad \eta=\frac{2 \beta}{1+\alpha^{2}+\beta^{2}}, \quad \zeta=\frac{-1+\alpha^{2}+\beta^{2}}{1+\alpha^{2}+\beta^{2}},
$$

where

$$
\frac{a}{b}=\alpha+\sqrt{-1} \beta
$$

Therefore for $g_{i}$, we get the sets $B\left(g_{i}\right)(1 \leq i \leq 3)$ which is written in the theorem above.

\section{The Lefschetz elements for $G(2 n, n, 2)$}

We recall that $G(2 n, n, 2)$ of order $8 n$ contains a binary dihedral group of order $4 n$ as a subgroup of index 2. In this section we give a characterization of the strong (weak) Lefschetz element of the coinvariant algebras of $G(2 n, n, 2)$.

Let $G=G(2 n, n, 2)$ :

$$
G=\left\langle\left(\begin{array}{ll}
0 & 1 \\
1 & 0
\end{array}\right),\left(\begin{array}{cc}
-1 & 0 \\
0 & 1
\end{array}\right),\left(\begin{array}{cc}
\theta & 0 \\
0 & \theta^{-1}
\end{array}\right)\right\rangle, \quad\left(\theta=e^{\frac{\pi i}{n}}\right) .
$$

The invariant algebra of $G$ is

$$
S(V)^{G}=\mathbf{C}\left[X^{2 n}+Y^{2 n}, X^{2} Y^{2}\right] .
$$

By (3) in 3.2 and Table 1, we have

$$
\begin{aligned}
P_{S(V)_{G}}(t) & =\frac{\left(1-t^{4}\right)\left(1-t^{2 n}\right)}{(1-t)^{2}} \\
& =1+2 t+3 t^{2}+4 t^{3}+\cdots+4 t^{2 n-1}+3 t^{2 n}+2 t^{2 n+1}+t^{2 n+2} .
\end{aligned}
$$

We choose a basis of $S(V)_{G}$

$$
\begin{aligned}
\left\{1, x, y, x^{2}, x y, y^{2}, x^{3}, x^{2} y, x y^{2}, y^{3},\right. & \ldots, x^{2 n-1}, x^{2 n-2} y, x y^{2 n-2}, y^{2 n-1}, \\
& \left.x^{2 n}, x^{2 n-1} y, x y^{2 n-1}, x^{2 n} y, x y^{2 n}, x^{2 n+1} y\right\} .
\end{aligned}
$$

THEOREM 4. (1) The element $l=a x+b y \in S_{1}(V)_{G}$ is the strong Lefschetz element if and only if $\prod_{i=1}^{4} g_{i} \neq 0$, where $g_{i}$ are the polynomials in $a, b$ given by $g_{1}(a, b)=a, g_{2}(a, b)=b, g_{3}(a, b)=a^{2 n}-b^{2 n}, g_{4}(a, b)=4 n^{2} a^{2 n} b^{2 n}+\left(a^{2 n}-b^{2 n}\right)^{2}$.

(See the end of $\$ 3$ for the notation)

(2) The element $l=a x+b y \in S_{1}(V)_{G}$ is the weak Lefschetz element if and only if $a \neq 0$ and $b \neq 0$. 
(3) We have the inverse images of $t\left(g_{i}\right)$ under the stereographic projection as follows (see the end of $\$ 3$ for the notation) :

$$
\begin{aligned}
& B\left(g_{1}\right)=\{(0,0,-1)\}, \\
& B\left(g_{2}\right)=\{(0,0,1)\}, \\
& B\left(g_{3}\right)=\left\{\left(\cos \frac{\pi k}{n}, \sin \frac{\pi k}{n}, 0\right)\right\},(k=0, \ldots, 2 n-1) .
\end{aligned}
$$

We take an $n$-sided regular polygon on XY-plane. If we put a vertex of the regular polygon on $(1,0,0)$, the sets $B\left(g_{i}\right)$ are given as follows :

(i) $B\left(g_{1}\right) \cup B\left(g_{2}\right)$ is the set of points corresponding to the centers of the faces of the regular polygon.

(ii) $B\left(g_{3}\right)$ is the set of $n$ vertices of the regular polygon and $n$ points corresponding to the centers of the edges of the regular polygon.

ProOF. (1) Since $I=\left(X^{2 n}+Y^{2 n}, X^{2} Y^{2}\right)$, we have

$$
x^{2} y^{2}=0, \quad x^{2 n}+y^{2 n}=0 .
$$

By (5.1), the matrix representation of $\sigma_{0}, \sigma_{1}, \sigma_{2}, \sigma_{i}(3 \leq i \leq n+1)$ with respect to the basis (5.0) are

$$
\begin{aligned}
& \left((2 n+2) a b\left(a^{2 n}-b^{2 n}\right)\right),\left(\begin{array}{cc}
2 n a^{2 n-1} b & a^{2 n}-b^{2 n} \\
-a^{2 n}+b^{2 n} & 2 n a b^{2 n-1}
\end{array}\right), \\
& \left(\begin{array}{ccc}
a^{2 n-2} & 0 & -b^{2 n-2} \\
(2 n-2) a^{2 n-3} b & a^{2 n-2} & 0 \\
0 & b^{2 n-2} & (2 n-2) a b^{2 n-3}
\end{array}\right), \\
& \left(\begin{array}{cccc}
a^{2 n+2-2 i} & 0 & 0 & 0 \\
(2 n+2-2 i) a^{2 n+1-2 i} b & a^{2 n+2-2 i} & 0 & 0 \\
0 & 0 & b^{2 n+2-2 i} & (2 n+2-2 i) a b^{2 n+1-2 i} \\
0 & 0 & 0 & b^{2 n+2-2 i}
\end{array}\right)
\end{aligned}
$$

respectively. Thus we get (1).

(2) By (5.1), the matrix representation of $\tau_{0}, \tau_{1}, \tau_{2}, \tau_{i}(3 \leq i \leq 2 n-2), \tau_{2 n-1}, \tau_{2 n}, \tau_{2 n+1}$ with respect to the basis (5.0) are

$$
\left(\begin{array}{l}
a \\
b
\end{array}\right), \quad\left(\begin{array}{ll}
a & 0 \\
b & a \\
0 & b
\end{array}\right), \quad\left(\begin{array}{lll}
a & 0 & 0 \\
b & a & 0 \\
0 & b & a \\
0 & 0 & b
\end{array}\right), \quad\left(\begin{array}{cccc}
a & 0 & 0 & 0 \\
b & a & 0 & 0 \\
0 & 0 & b & a \\
0 & 0 & 0 & b
\end{array}\right), \quad\left(\begin{array}{cccc}
a & 0 & 0 & -b \\
b & a & 0 & 0 \\
0 & 0 & b & a
\end{array}\right)
$$




$$
\left(\begin{array}{ccc}
b & a & 0 \\
-a & 0 & b
\end{array}\right), \quad\left(\begin{array}{ll}
a & -b
\end{array}\right)
$$

respectively. Thus we get (2).

(3) For a homogeneous polynomial $g_{i}$, the points $(a: b) \in t\left(g_{i}\right)$ are as follows:

$$
\begin{gathered}
t\left(g_{1}\right)=\{(0: 1)\}, \\
t\left(g_{2}\right)=\{(1: 0)\}, \\
t\left(g_{3}\right)=\left\{\left(\theta^{k}: 1\right) \mid k=0, \ldots, 2 n-1\right\}, \theta=e^{\frac{\pi \sqrt{-1}}{n}} .
\end{gathered}
$$

We identify the sphere $S^{2}$ with $\mathbf{C} \cup\{\infty\}$ by the stereographic projection $p$ (see the relations $(*)$ of the end of $\S 4)$.

Therefore for $g_{i}$, we get the sets $B\left(g_{i}\right)(1 \leq i \leq 3)$ which is written in the theorem above.

\section{The Lefschetz elements for $G_{12}$}

We recall that the group $G_{12}$ of order 48 contains a binary tetrahedral group of order 24 as a subgroup of index 2 . In this section we give a characterization of the strong Lefschetz element of the coinvariant algebras of $G_{12}$.

Let $G=G_{12}$ :

$$
G=\left\langle\frac{1}{\sqrt{2}}\left(\begin{array}{ll}
-1 & i \\
-i & 1
\end{array}\right), \frac{1}{\sqrt{2}}\left(\begin{array}{cc}
\varepsilon & \varepsilon \\
\varepsilon^{3} & \varepsilon^{7}
\end{array}\right)\right\rangle, \quad\left(\varepsilon=e^{\frac{2 \pi i}{8}}\right) .
$$

The invariant algebra of $G$ is

$$
S(V)^{G}=\mathbf{C}\left[X Y\left(X^{4}-Y^{4}\right), X^{8}+14 X^{4} Y^{4}+Y^{8}\right] .
$$

By (3) in 3.2 and Table 1, we have

$$
\begin{aligned}
P_{S(V)_{G}}(t) & =\frac{\left(1-t^{6}\right)\left(1-t^{8}\right)}{(1-t)^{2}} \\
& =1+2 t+3 t^{2}+4 t^{3}+5 t^{4}+6 t^{5}+6 t^{6}+6 t^{7}+5 t^{8}+4 t^{9}+3 t^{10}+2 t^{11}+t^{12} .
\end{aligned}
$$

We choose a basis of $S(V)_{G}$

$$
\begin{array}{r}
\left\{1, x, y, x^{2}, x y, y^{2}, x^{3}, x^{2} y, x y^{2}, y^{3}, x^{4}, x^{3} y, x^{2} y^{2}, x y^{3}, y^{4}\right. \\
x^{5}, x^{4} y, x^{3} y^{2}, x^{2} y^{3}, x y^{4}, y^{5}, x^{6}, x^{4} y^{2}, x^{3} y^{3}, x^{2} y^{4}, x y^{5}, y^{6} \\
x^{7}, x^{5} y^{2}, x^{4} y^{3}, x^{3} y^{4}, x^{2} y^{5}, y^{7}, x^{8}, x^{5} y^{3}, x^{3} y^{5}, x^{2} y^{6}, y^{8}, \\
\left.x^{9}, x^{6} y^{3}, x^{3} y^{6}, y^{9}, x^{10}, x^{3} y^{7}, y^{10}, x^{11}, y^{11}, y^{12}\right\}
\end{array}
$$


THEOREM 5. (1) The element $l=a x+b y \in S_{1}(V)_{G}$ is the strong Lefschetz element if and only if $\prod_{i=1}^{12} g_{i} \neq 0$, where $g_{i}$ are the polynomials in $a, b$ given by

$$
\begin{aligned}
g_{1} & =a, \quad g_{2}=b, \quad g_{3}=a-b, \quad g_{4}=a+b, \quad g_{5}=a^{2}+b^{2}, \\
g_{6} & =a^{2}+2 a b-b^{2}, \quad g_{7}=a^{2}-2 a b-b^{2}, \quad g_{8}=a^{4}+b^{4}, \quad g_{9}=a^{4}+6 a^{2} b^{2}+b^{4}, \\
g_{10} & =a^{4}+2 a^{3} b+2 a^{2} b^{2}-2 a b^{3}+b^{4}, \quad g_{11}=a^{4}-2 a^{3} b+2 a^{2} b^{2}+2 a b^{3}+b^{4}, \\
g_{12} & =5 a^{24}+642 a^{20} b^{4}+1227 a^{16} b^{8}+16732 a^{12} b^{12}+1227 a^{8} b^{16}+642 a^{4} b^{20}+5 b^{24} .
\end{aligned}
$$

(See the end of $\$ 3$ for the notation.)

(2) We have the inverse images of $t\left(g_{i}\right)(1 \leq i \leq 11)$ under the stereographic projection as follows (see the end of $\$ 3$ for the notation) :

$$
\begin{aligned}
B\left(g_{1}\right) & =\{(0,0,-1)\}, \\
B\left(g_{2}\right) & =\{(0,0,1)\}, \\
B\left(g_{3}\right) & =\{(1,0,0)\}, \\
B\left(g_{4}\right) & =\{(-1,0,0)\}, \\
B\left(g_{5}\right) & =\{(0,1,0),(0,-1,0)\}, \\
B\left(g_{6}\right) & =\left\{\left(\frac{1}{\sqrt{2}}, 0,-\frac{1}{\sqrt{2}}\right),\left(-\frac{1}{\sqrt{2}}, 0, \frac{1}{\sqrt{2}}\right)\right\}, \\
B\left(g_{7}\right) & =\left\{\left(\frac{1}{\sqrt{2}}, 0, \frac{1}{\sqrt{2}}\right),\left(-\frac{1}{\sqrt{2}}, 0,-\frac{1}{\sqrt{2}}\right)\right\}, \\
B\left(g_{8}\right) & =\left\{\left(\frac{1}{\sqrt{2}}, \frac{1}{\sqrt{2}}, 0\right),\left(-\frac{1}{\sqrt{2}},-\frac{1}{\sqrt{2}}, 0\right),\left(-\frac{1}{\sqrt{2}}, \frac{1}{\sqrt{2}}, 0\right),\left(\frac{1}{\sqrt{2}},-\frac{1}{\sqrt{2}}, 0\right)\right\}, \\
B\left(g_{9}\right) & =\left\{\left(0, \frac{1}{\sqrt{2}}, \frac{1}{\sqrt{2}}\right),\left(0,-\frac{1}{\sqrt{2}}, \frac{1}{\sqrt{2}}\right),\left(0, \frac{1}{\sqrt{2}},-\frac{1}{\sqrt{2}}\right),\left(0,-\frac{1}{\sqrt{2}},-\frac{1}{\sqrt{2}}\right)\right\}, \\
B\left(g_{10}\right) & =\left\{\left(\frac{\sqrt{3}}{3},-\frac{\sqrt{3}}{3},-\frac{\sqrt{3}}{3}\right),\left(-\frac{\sqrt{3}}{3}, \frac{\sqrt{3}}{3}, \frac{\sqrt{3}}{3}\right),\left(\frac{\sqrt{3}}{3}, \frac{\sqrt{3}}{3},-\frac{\sqrt{3}}{3}\right),\left(-\frac{\sqrt{3}}{3},-\frac{\sqrt{3}}{3}, \frac{\sqrt{3}}{3}\right)\right\}, \\
B\left(g_{11}\right) & =\left\{\left(\frac{\sqrt{3}}{3},-\frac{\sqrt{3}}{3}, \frac{\sqrt{3}}{3}\right),\left(-\frac{\sqrt{3}}{3}, \frac{\sqrt{3}}{3},-\frac{\sqrt{3}}{3}\right),\left(\frac{\sqrt{3}}{3}, \frac{\sqrt{3}}{3}, \frac{\sqrt{3}}{3}\right),\left(-\frac{\sqrt{3}}{3},-\frac{\sqrt{3}}{3},-\frac{\sqrt{3}}{3}\right)\right\} .
\end{aligned}
$$

There are, in a cube, two tetrahedra whose edges are the diagonals of the faces of the cube. The dual of the cube is the octahedron. Now if we take an octahedron with vertices $( \pm 1,0,0),(0, \pm 1,0),(0,0, \pm 1)$ in $R^{3}$, the sets $B\left(g_{i}\right)$ are given as follows :

(i) The sets of $B\left(g_{1}\right), B\left(g_{2}\right), B\left(g_{3}\right), B\left(g_{4}\right), B\left(g_{5}\right)$ are the six points of $S^{2}$ corresponding to the centers of the edges of the tetrahedron (or the six vertices of the octahedron).

(ii) The sets of $B\left(g_{6}\right), B\left(g_{7}\right), B\left(g_{8}\right), B\left(g_{9}\right)$ are the twelve points of $S^{2}$ corresponding to the centers of the edges of the octahedron.

(iii) The sets of $B\left(g_{10}\right), B\left(g_{11}\right)$ are the four points of $S^{2}$ corresponding to the vertices of the tetrahedron and the four points of $S^{2}$ corresponding to the centers of 
the faces of the tetrahedron (or the eight points of $S^{2}$ corresponding to the centers of the faces of the octahedron).

Proof. (1) Since $I_{G}=\left(X Y\left(X^{4}-Y^{4}\right), X^{8}+14 X^{4} Y^{4}+Y^{8}\right)$, we have

$$
\begin{gathered}
x y\left(x^{4}-y^{4}\right)=0, \\
x^{8}+14 x^{4} y^{4}+y^{8}=0 .
\end{gathered}
$$

For later use, we prepare the following relations. By (6.1), we have

$$
x^{9} y=\left(x y^{5}\right) x^{4}=x^{5} y^{5}=\left(x y^{5}\right) y^{4}=x y^{9} .
$$

Moreover we have

$$
\begin{aligned}
x^{5} y^{5} & =-\frac{1}{14} x y\left(x^{8}+y^{8}\right)(\text { by }(6.2)) \\
& =-\frac{1}{7} x^{5} y^{5} .(\text { by }(6.3))
\end{aligned}
$$

Thus we have $x^{5} y^{5}=0$. Therefore

$$
x^{5} y^{5}=x^{9} y=x y^{9}=0 .
$$

By (6.1), (6.4), we have

$$
x^{i} y^{j}=x^{i-4} y^{j+4}, \quad i \geq j \geq 1
$$

and

$$
x^{i} y^{j}=0, \quad i \geq 9, j \geq 1 \text { or } i \geq 1, j \geq 9 .
$$

By (6.2), (6.5), we have

$$
\begin{array}{rlrl}
x^{8} y^{j}=x^{4} y^{j+4}=-\frac{1}{15} y^{8+j}, & & 1 \leq j \leq 4, \\
x^{i} y^{8}=x^{i+4} y^{4}=-\frac{1}{15} x^{8+i}, & 1 \leq i \leq 4 .
\end{array}
$$

(i) By $(6.5), \ldots,(6.8)$, we have

$$
\begin{aligned}
x^{12}=y^{12}, x^{11} y= & x^{10} y^{2}=x^{9} y^{3}=x^{7} y^{5}=x^{6} y^{6}=x^{5} y^{7}=x^{3} y^{9} \\
& =x^{2} y^{10}=x y^{11}=0, x^{8} y^{4}=x^{4} y^{8}=-\frac{1}{15} x^{12}=-\frac{1}{15} y^{12} .
\end{aligned}
$$

For $i=0$, by (6.9), the matrix representation of $\sigma_{0}$ with respect to the basis $(6,0)$ is regular if and only if $a^{12}-33 a^{8} b^{4}-33 a^{4} b^{8}+b^{12} \neq 0$, i.e. $\left(a^{2}+2 a b-b^{2}\right)\left(a^{2}-2 a b-\right.$ $\left.b^{2}\right)\left(a^{4}-b^{4}\right)\left(a^{4}+6 a^{2} b^{2}+b^{4}\right) \neq 0$. 
(ii) By $(6.5), \ldots,(6.8)$, we have

$$
\begin{aligned}
& x^{10} y=x^{9} y^{2}=x^{6} y^{5}=x^{5} y^{6}=x^{2} y^{9}=x y^{10}=0, \\
& x^{8} y^{3}=x^{4} y^{7}=-\frac{1}{15} y^{11}, x^{7} y^{4}=x^{3} y^{8}=-\frac{1}{15} y^{12} .
\end{aligned}
$$

For $i=1$, by (6.10), the matrix representation of $\sigma_{1}$ with respect to the basis $(6,0)$ is

$$
\left(\begin{array}{cc}
a^{10}-14 a^{6} b^{4}-3 a^{2} b^{8} & -8 a^{3} b^{3}\left(a^{4}+b^{4}\right) \\
-8 a^{3} b^{3}\left(a^{4}+b^{4}\right) & -3 a^{8} b^{2}-14 a^{4} b^{6}+b^{10}
\end{array}\right) .
$$

Therefore $\sigma_{1}$ is bijective if and only if $-3 a^{2} b^{2}(a-b)^{2}(a+b)^{2}\left(a^{2}+b^{2}\right)^{2}\left(a^{4}+2 a^{3} b+\right.$ $\left.2 a^{2} b^{2}-2 a b^{3}+b^{4}\right)\left(a^{4}-2 a^{3} b+2 a^{2} b^{2}+2 a b^{3}+b^{4}\right) \neq 0$.

(iii) By (6.5), .., (6.8), we have

$$
\begin{aligned}
& x^{5} y^{5}=x^{9} y=x y^{9}=0, \quad x^{7} y^{3}=x^{3} y^{7}, \\
& x^{8} y^{2}=x^{4} y^{6}=-\frac{1}{15} y^{10}, \quad x^{6} y^{4}=x^{2} y^{8}=-\frac{1}{15} x^{10} .
\end{aligned}
$$

For $i=2$, by (6.11), the matrix representation of $\sigma_{2}$ with respect to the basis $(6,0)$ is

$$
\left(\begin{array}{ccc}
\frac{1}{15}\left(15 a^{8}-70 a^{4} b^{4}-b^{8}\right) & -\frac{8}{15} a b\left(7 a^{4} b^{2}+b^{6}\right) & -\frac{28}{15} a^{2} b^{2}\left(a^{4}+b^{4}\right) \\
8 a b\left(7 a^{4} b^{2}+b^{6}\right) & 28 a^{2} b^{2}\left(a^{4}+b^{4}\right) & 8 a b\left(7 a^{2} b^{4}+a^{6}\right) \\
-\frac{28}{15} a^{2} b^{2}\left(a^{4}+b^{4}\right) y^{10} & -\frac{8}{15} a b\left(7 a^{2} b^{4}+a^{6}\right) & -\frac{1}{15}\left(a^{8}+70 a^{4} b^{4}-15 b^{8}\right)
\end{array}\right) .
$$

Therefore $\sigma_{2}$ is bijective if and only if $\frac{12}{5} a^{2} b^{2}(a-b)^{2}(a+b)^{2}\left(a^{2}+b^{2}\right)^{2}\left(a^{2}+2 a b-\right.$ $\left.b^{2}\right)\left(a^{2}-2 a b-b^{2}\right)\left(a^{4}+b^{4}\right)\left(a^{4}+6 a^{2} b^{2}+b^{4}\right) \neq 0$.

(iv) By (6.5), (6.7), (6.8), we have

$$
\begin{aligned}
& x^{7} y^{2}=x^{3} y^{6}, \quad x^{6} y^{3}=x^{2} y^{7}, \\
& x^{8} y=x^{4} t^{5}=-\frac{1}{15} y^{9}, \quad x^{5} y^{4}=x y^{8}=-\frac{1}{15} x^{9} .
\end{aligned}
$$

For $i=3$, by (6.12), the matrix representation of $\sigma_{3}$ with respect to the basis $(6,0)$ is

$$
\left(\begin{array}{cccc}
a^{2}\left(a^{4}-b^{4}\right) & -\frac{4}{3} a^{3} b^{3} & -\frac{1}{15} b^{2}\left(15 a^{4}+b^{4}\right) & -\frac{2}{5} a b\left(a^{4}+b^{4}\right) \\
20 a^{3} b^{3} & b^{2}\left(15 a^{4}+b^{4}\right) & 6 a b\left(a^{4}+b^{4}\right) & a^{2}\left(a^{4}+15 b^{4}\right) \\
b^{2}\left(15 a^{4}+b^{4}\right) & 6 a b\left(a^{4}+b^{4}\right) & a^{2}\left(a^{4}+15 b^{4}\right) & 20 a^{3} b^{3} \\
-\frac{2}{5} a b\left(a^{4}+b^{4}\right) & -\frac{1}{15} a^{2}\left(a^{4}+15 b^{4}\right) & -\frac{4}{3} a^{3} b^{3} & b^{2}\left(-a^{4}+b^{4}\right)
\end{array}\right) .
$$

Therefore $\sigma_{3}$ is bijective if and only if $\frac{1}{71}\left(5 a^{24}+642 a^{20} b^{4}+1227 a^{16} b^{8}+\right.$ $\left.16732 a^{12} b^{12}+1227 a^{8} b^{16}+642 a^{4} b^{20}+5 b^{24}\right) \neq 0$.

(v) By (6.5), we have

$$
x^{7} y=x^{3} y^{5}, \quad x^{6} y^{2}=x^{2} y^{6}, \quad x^{5} y^{3}=x y^{7} .
$$


For $i=4$, by (6.13), the matrix representation of $\sigma_{4}$ with respect to the basis $(6,0)$ is

$$
\left(\begin{array}{ccccc}
a^{4}-\frac{1}{14} b^{4} & -\frac{2}{7} a b^{3} & -\frac{3}{7} a^{2} b^{2} & -\frac{2}{7} a^{3} b & -\frac{1}{14} a^{4} \\
4 a b^{3} & 6 a^{2} b^{2} & 4 a^{3} b & a^{4}+b^{4} & 4 a b^{3} \\
4 a^{3} b & a^{4}+b^{4} & 4 a b^{3} & 6 a^{2} b^{2} & 4 a^{3} b \\
6 a^{2} b^{2} & 4 a^{3} b & a^{4}+b^{4} & 4 a b^{3} & 6 a^{2} b^{2} \\
-\frac{1}{14} b^{4} & -\frac{2}{7} a b^{3} & -\frac{3}{7} a^{2} b^{2} & -\frac{2}{7} a^{3} b & -\frac{1}{14} a^{4}+b^{4}
\end{array}\right)
$$

Therefore $\sigma_{4}$ is bijective if and only if $-\frac{1}{14}\left(a^{2}+2 a b-b^{2}\right)\left(a^{2}-2 a b-b^{2}\right)\left(a^{4}+b^{4}\right)\left(a^{4}+\right.$ $\left.6 a^{2} b^{2}+b^{4}\right)\left(a^{4}+2 a^{3} b+2 a^{2} b^{2}-2 a b^{3}+b^{4}\right)\left(a^{4}-2 a^{3} b+2 a^{2} b^{2}+2 a b^{3}+b^{4}\right) \neq 0$.

(vi) By (6.5), we have

$$
x^{6} y=x^{2} y^{5}, x y^{6}=x^{5} y^{2}
$$

For $i=5$, by (6.14), the matrix representation of $\sigma_{5}$ with respect to the basis $(6,0)$ is

$$
\left(\begin{array}{cccccc}
a^{2} & 0 & 0 & 0 & 0 & 0 \\
b^{2} & 2 a b & a^{2} & 0 & b^{2} & 2 a b \\
0 & b^{2} & 2 a b & a^{2} & 0 & 0 \\
0 & 0 & b^{2} & 2 a b & a^{2} & 0 \\
2 a b & a^{2} & 0 & b^{2} & 2 a b & a^{2} \\
0 & 0 & 0 & 0 & 0 & b^{2}
\end{array}\right)
$$

Therefore $\sigma_{5}$ is bijective if and only if $a^{2} b^{2}(a-b)^{2}(a+b)^{2}\left(a^{2}+b^{2}\right)^{2} \neq 0$.

(vii) For $i=6, \sigma_{6}$ is bijective obviously.

From (i), ..., (vii), we get (1).

(2) For a homogeneous polynomial $g_{i}$, the points $(a: b) \in t\left(g_{i}\right)$ are as follows:

$$
\begin{aligned}
& t\left(g_{1}\right)=\{(0: 1)\}, \\
& t\left(g_{2}\right)=\{(1: 0)\}, \\
& t\left(g_{3}\right)=\{(1: 1)\}, \\
& t\left(g_{4}\right)=\{(-1: 1)\}, \\
& t\left(g_{5}\right)=\{( \pm i: 1)\}, \\
& t\left(g_{6}\right)=\{(-1 \pm \sqrt{2}: 1)\}, \\
& t\left(g_{7}\right)=\{(1 \pm \sqrt{2}: 1)\}, \\
& t\left(g_{8}\right)=\left\{\left( \pm(-1)^{\frac{1}{4}}: 1\right),\left( \pm(-1)^{\frac{1}{4}} i: 1\right)\right\}, \\
& t\left(g_{9}\right)=\{( \pm(\sqrt{2}+1) i: 1),( \pm(\sqrt{2}-1) i: 1)\},
\end{aligned}
$$




$$
\begin{aligned}
& t\left(g_{10}\right)=\left\{\left(\frac{1}{2}(-1+i \pm \sqrt{-6 i}): 1\right),\left(\frac{1}{2}(-1-i \pm \sqrt{6 i}): 1\right)\right\}, \\
& t\left(g_{11}\right)=\left\{\left(\frac{1}{2}(1-i \pm \sqrt{-6 i}): 1\right),\left(\frac{1}{2}(1+i \pm \sqrt{6 i}): 1\right)\right\} .
\end{aligned}
$$

We identify the sphere $S^{2}$ with $\mathbf{C} \cup\{\infty\}$ by the stereographic projection $p$ (See the relations $(*)$ of the end of $\S 4)$.

Therefore for $g_{i}$, we get the sets $B\left(g_{i}\right)(1 \leq i \leq 11)$ which is written in the theorem above.

\section{The Lefschetz elements for $G_{13}$}

We recall that the group $G_{13}$ of order 96 contains a binary octahedral group of order 48 as a subgroup of index 2 . In this section we give a characterization of the strong Lefschetz element of the coinvariant algebras of $G_{13}$.

Let $G=G_{13}$ :

$$
G=\left\langle\frac{1}{\sqrt{2}}\left(\begin{array}{ll}
-1 & i \\
-i & 1
\end{array}\right), \frac{1}{\sqrt{2}}\left(\begin{array}{cc}
\varepsilon^{3} & \varepsilon^{3} \\
\varepsilon^{5} & \varepsilon
\end{array}\right)\right\rangle,\left(\varepsilon=e^{\frac{2 \pi i}{8}}\right)
$$

The invariant algebra of $G$ is

$$
S(V)^{G}=\mathbf{C}\left[X^{8}+14 X^{4} Y^{4}+Y^{8}, X^{10} Y^{2}-2 X^{6} Y^{6}+X^{2} Y^{10}\right] .
$$

By (3) in 3.2 and Table 1, we have

$$
\begin{aligned}
P_{S(V)_{G}}(t) & =\frac{\left(1-t^{8}\right)\left(1-t^{12}\right)}{(1-t)^{2}} \\
& =1+2 t+3 t^{2}+4 t^{3}+5 t^{4}+6 t^{5}+7 t^{6}+8 t^{7}+\cdots+8 t^{11}+7 t^{12}+6 t^{13} \\
& +5 t^{14}+4 t^{15}+3 t^{16}+2 t^{17}+t^{18} .
\end{aligned}
$$

We choose a basis of $S(V)_{G}$

$$
\begin{gathered}
\left\{1, x, y, x^{2}, x y, y^{2}, x^{3}, x^{2} y, x y^{2}, y^{3}, x^{4}, x^{3} y, x^{2} y^{2}, x y^{3}, y^{4}, x^{5}, x^{4} y, x^{3} y^{2}, x^{2} y^{3}, x y^{4},\right. \\
y^{5}, x^{6}, x^{5} y, x^{4} y^{2}, x^{3} y^{3}, x^{2} y^{4}, x y^{5}, y^{6}, x^{7}, x^{6} y, x^{5} y^{2}, x^{4} y^{3}, x^{3} y^{4}, x^{2} y^{5}, x y^{6}, y^{7}, \\
x^{7} y, x^{6} y^{2}, x^{5} y^{3}, x^{4} y^{4}, x^{3} y^{5}, x^{2} y^{6}, x y^{7}, y^{8}, x^{8} y, x^{7} y^{2}, x^{6} y^{3}, x^{5} y^{4}, x^{4} y^{5}, x^{3} y^{6}, x^{2} y^{7}, x y^{8}, \\
x^{8} y^{2}, x^{7} y^{3}, x^{6} y^{4}, x^{5} y^{5}, x^{4} y^{6}, x^{3} y^{7}, x^{2} y^{8}, x y^{9}, x^{9} y^{2}, x^{8} y^{3}, x^{7} y^{4}, x^{6} y^{5}, x^{5} y^{6}, x^{4} y^{7}, x^{3} y^{8}, \\
x^{2} y^{9}, x^{9} y^{3}, x^{8} y^{4}, x^{7} y^{5}, x^{5} y^{7}, x^{4} y^{8}, x^{3} y^{9}, x^{2} y^{10}, x^{10} y^{3}, x^{9} y^{4}, x^{8} y^{5}, x^{5} y^{8}, x^{4} y^{9}, x^{3} y^{10}, \\
x^{10} y^{4}, x^{9} y^{5}, x^{5} y^{9}, x^{4} y^{10}, x^{3} y^{11}, x^{11} y^{4}, x^{10} y^{5}, x^{5} y^{10}, x^{4} y^{11}, x^{11} y^{5}, x^{5} y^{11}, x^{4} y^{12}, \\
\left.x^{12} y^{5}, x^{5} y^{12}, x^{13} y^{5}\right\}
\end{gathered}
$$


THEOREM 6. (1) The element $l=a x+b y \in S_{1}(V)_{G}$ is the strong Lefschetz element if and only if $\prod_{i=1}^{17} g_{i} \neq 0$, where $g_{i}$ are the polynomials in $a, b$ given by

$$
\begin{aligned}
g_{1}= & a, \quad g_{2}=b, \quad g_{3}=a-b, \quad g_{4}=a+b, \quad g_{5}=a^{2}+b^{2}, \\
g_{6}= & a^{2}+2 a b-b^{2}, \quad g_{7}=a^{2}-2 a b-b^{2}, \quad g_{8}=a^{4}+b^{4}, g_{9}=a^{4}+6 a^{2} b^{2}+b^{4}, \\
g_{10}= & a^{4}+2 a^{3} b+2 a^{2} b^{2}-2 a b^{3}+b^{4}, \quad g_{11}=a^{4}-2 a^{3} b+2 a^{2} b^{2}+2 a b^{3}+b^{4}, \\
g_{12}= & a^{24}+366 a^{20} b^{4}-705 a^{16} b^{8}+4772 a^{12} b^{12}-705 a^{8} b^{16}+366 a^{4} b^{20}+b^{24}, \\
g_{13}= & 2 a^{24}+3 a^{20} b^{4}+1506 a^{16} b^{8}+5170 a^{12} b^{12}+1506 a^{8} b^{16}+3 a^{4} b^{20}+2 b^{24}, \\
g_{14}= & 2 a^{24}-637 a^{20} b^{4}+4066 a^{16} b^{8}+1330 a^{12} b^{12}+4066 a^{8} b^{16}-637 a^{4} b^{20}+2 b^{24}, \\
g_{15}= & a^{24}-470 a^{20} b^{4}+2639 a^{16} b^{8}-244 a^{12} b^{12}+2639 a^{8} b^{16}-470 a^{4} b^{20}+b^{24}, \\
g_{16}= & 4 a^{48}-468 a^{44} b^{4}+39633 a^{40} b^{8}-590924 a^{36} b^{12}+3414840 a^{32} b^{16} \\
& +16202208 a^{28} b^{20}+28978278 a^{24} b^{24}+16202208 a^{20} b^{28}+3414840 a^{16} b^{32} \\
& -590924 a^{12} b^{36}+39633 a^{8} b^{40}-468 a^{4} b^{44}+4 b^{48}, \\
g_{17}= & a^{48}+4312 a^{44} b^{4}+348186 a^{40} b^{8}+392504 a^{36} b^{12}+8766479 a^{32} b^{16} \\
& -37985808 a^{28} b^{20}+73725868 a^{24} b^{24}-37985808 a^{20} b^{28}+8766479 a^{16} b^{32} \\
& +392504 a^{12} b^{36}+348186 a^{8} b^{40}+4312 a^{4} b^{44}+b^{48}
\end{aligned}
$$

(See the end of $\$ 3$ for the notation.)

(2) We have the inverse images of $\left(g_{i}\right)(1 \leq i \leq 11)$ under the stereographic projection as follows (see the end of $\S 3$ for the notation) :

$$
\begin{aligned}
& B\left(g_{1}\right)=\{(0,0,-1)\}, \\
& B\left(g_{2}\right)=\{(0,0,1)\}, \\
& B\left(g_{3}\right)=\{(1,0,0)\}, \\
& B\left(g_{4}\right)=\{(-1,0,0)\}, \\
& B\left(g_{5}\right)=\{(0,1,0),(0,-1,0)\}, \\
& B\left(g_{6}\right)=\left\{\left(\frac{1}{\sqrt{2}}, 0,-\frac{1}{\sqrt{2}}\right),\left(-\frac{1}{\sqrt{2}}, 0, \frac{1}{\sqrt{2}}\right)\right\}, \\
& B\left(g_{7}\right)=\left\{\left(\frac{1}{\sqrt{2}}, 0, \frac{1}{\sqrt{2}}\right),\left(-\frac{1}{\sqrt{2}}, 0,-\frac{1}{\sqrt{2}}\right)\right\}, \\
& B\left(g_{8}\right)=\left\{\left(\frac{1}{\sqrt{2}}, \frac{1}{\sqrt{2}}, 0\right),\left(-\frac{1}{\sqrt{2}},-\frac{1}{\sqrt{2}}, 0\right),\left(-\frac{1}{\sqrt{2}}, \frac{1}{\sqrt{2}}, 0\right),\left(\frac{1}{\sqrt{2}},-\frac{1}{\sqrt{2}}, 0\right)\right\}, \\
& B\left(g_{9}\right)=\left\{\left(0, \frac{1}{\sqrt{2}}, \frac{1}{\sqrt{2}}\right),\left(0,-\frac{1}{\sqrt{2}}, \frac{1}{\sqrt{2}}\right),\left(0, \frac{1}{\sqrt{2}},-\frac{1}{\sqrt{2}}\right),\left(0,-\frac{1}{\sqrt{2}},-\frac{1}{\sqrt{2}}\right)\right\}, \\
& B\left(g_{10}\right)=\left\{\left(\frac{\sqrt{3}}{3},-\frac{\sqrt{3}}{3},-\frac{\sqrt{3}}{3}\right),\left(-\frac{\sqrt{3}}{3}, \frac{\sqrt{3}}{3}, \frac{\sqrt{3}}{3}\right),\left(\frac{\sqrt{3}}{3}, \frac{\sqrt{3}}{3},-\frac{\sqrt{3}}{3}\right),\left(-\frac{\sqrt{3}}{3},-\frac{\sqrt{3}}{3}, \frac{\sqrt{3}}{3}\right)\right\},
\end{aligned}
$$


$B\left(g_{11}\right)=\left\{\left(\frac{\sqrt{3}}{3},-\frac{\sqrt{3}}{3}, \frac{\sqrt{3}}{3}\right),\left(-\frac{\sqrt{3}}{3}, \frac{\sqrt{3}}{3},-\frac{\sqrt{3}}{3}\right),\left(\frac{\sqrt{3}}{3}, \frac{\sqrt{3}}{3}, \frac{\sqrt{3}}{3}\right),\left(-\frac{\sqrt{3}}{3},-\frac{\sqrt{3}}{3},-\frac{\sqrt{3}}{3}\right)\right\}$.

Now if we take the octahedron and the tetrahedron in $R^{3}$ in the same way as Theorem 5 (2), the sets $B\left(g_{i}\right)$ are given as follows :

(i) The sets of $B\left(g_{1}\right), B\left(g_{2}\right), B\left(g_{3}\right), B\left(g_{4}\right), B\left(g_{5}\right)$ are the six vertices of the octahedron (the six points of $S^{2}$ corresponding to the centers of the edges of the tetrahedron).

(ii) The sets of $B\left(g_{6}\right), B\left(g_{7}\right), B\left(g_{8}\right), B\left(g_{9}\right)$ are the twelve points of $S^{2}$ corresponding to the centers of the edges of the octahedron.

(iii) The sets of $B\left(g_{10}\right), B\left(g_{11}\right)$ are the eight points of $S^{2}$ corresponding to the centers of the faces of the octahedron (the four points of $S^{2}$ corresponding to the vertices of the tetrahedron and the four points of $S^{2}$ corresponding to the centers of the faces of the tetrahedron).

Proof. (1) Since $I_{G}=\left(X^{8}+14 X^{4} Y^{4}+Y^{8}, X^{10} Y^{2}-2 X^{6} Y^{6}+X^{2} Y^{10}\right)$, we have

$$
\begin{gathered}
x^{8}+14 x^{4} y^{4}+y^{8}=0, \\
x^{10} y^{2}-2 x^{6} y^{6}+x^{2} y^{10}=0 .
\end{gathered}
$$

For later use, we introduce the following relations. By (7.2), we have

$$
x^{6} y^{6}=\frac{1}{2}\left(x^{10} y^{2}+x^{2} y^{10}\right) .
$$

Since $x^{4} y^{4}=-\frac{1}{14}\left(x^{8}+y^{8}\right)$ by (7.1), we have

$$
x^{6} y^{6}=x^{2} y^{2}\left\{-\frac{1}{14}\left(x^{8}+y^{8}\right)\right\}=-\frac{1}{14}\left(x^{10} y^{2}+x^{2} y^{10}\right) \text {. }
$$

Therefore by (7.3), (7.4), we have

$$
x^{6} y^{6}=x^{10} y^{2}+x^{2} y^{10}=0 .
$$

By (7.5), we have

$$
x^{14} y^{2}=x^{4}\left(-x^{2} y^{10}\right)=0, \quad x^{2} y^{14}=y^{4}\left(-x^{10} y^{2}\right)=0 .
$$

Therefore by (7.5), (7.6), we have

$$
x^{i} y^{j}=0, \quad i, j \geq 6 \quad \text { or } \quad i \geq 14, j \geq 2 \quad \text { or } \quad i \geq 2, j \geq 14
$$

and

$$
x^{i} y^{j}=-x^{i-8} y^{j+8}, \quad i \geq 10, j \geq 2 .
$$

By (7.1), (7.7), we have

$$
x^{18}=y^{18}=0 .
$$


Also by (7.1), (7.7), (7.8), we have

$$
\begin{array}{ll}
x^{i} y^{j}=-14 x^{i-4} y^{j+4}, & i \geq 14, \\
x^{i} y^{j}=-14 x^{i+4} y^{j-4}, & j \geq 14 .
\end{array}
$$

and

$$
\begin{array}{ll}
x^{i} y^{j}=-14 x^{i-4} y^{j+4}-x^{i-8} y^{j+8}, & i \geq 8, \\
x^{i} y^{j}=-14 x^{i+4} y^{j-4}-x^{i+8} y^{j-8}, & j \geq 8 .
\end{array}
$$

(i) By (7.7), ., (7.10), we have

$$
\begin{array}{r}
x^{12} y^{6}=x^{11} y^{7}=x^{10} y^{8}=x^{9} y^{9}=x^{8} y^{10}=x^{7} y^{11}=x^{6} y^{12}=0 \\
x^{16} y^{2}=x^{15} y^{3}=x^{14} y^{4}=x^{4} y^{14}=x^{3} y^{15}=x^{2} y^{16}=0 \\
x^{18}=y^{18}=0, x^{17} y=-14 x^{13} y^{5}=14 x^{5} y^{13}=-x y^{17} .
\end{array}
$$

For $i=0$, by (7.12), the matrix representation of $\sigma_{0}$ with respect to the basis (7.0) is regular if and only if $-252 a b(a+b)(a-b)\left(a^{2}+b^{2}\right)\left(a^{2}-2 a b-b^{2}\right)\left(a^{2}+2 a b-\right.$ $\left.b^{2}\right)\left(a^{4}+b^{4}\right)\left(a^{4}+6 a^{2} b^{2}+b^{4}\right) \neq 0$.

(ii) By (7.7), (7.8), (7.10), we have

$$
\begin{aligned}
x^{11} y^{6} & =x^{10} y^{7}=x^{9} y^{8}=x^{8} y^{9}=x^{7} y^{10}=x^{6} y^{11}=0, \\
x^{15} y^{2} & =x^{14} y^{3}=x^{3} y^{14}=x^{2} y^{15}=0, \\
x^{17} & =-14 x^{13} y^{4}=14 x^{5} y^{12}=-x y^{16}, \\
y^{17} & =-14 x^{4} y^{13}=14 x^{12} y^{5}=-x^{16} y .
\end{aligned}
$$

For $i=1$, by (7.13), the matrix representation of $\sigma_{1}$ with respect to the basis (7.0) is

$$
\left(\begin{array}{cc}
-112 a^{3} b\left(2 a^{12}-39 a^{8} b^{4}+5 b^{12}\right) & -14\left(a^{16}-130 a^{12} b^{4}+130 a^{4} b^{12}-b^{16}\right) \\
14\left(a^{16}-130 a^{12} b^{4}+130 a^{4} b^{12}-b^{16}\right) & -112 a b^{3}\left(5 a^{12}-39 a^{4} b^{8}+2 b^{12}\right)
\end{array}\right) .
$$

Therefore $\sigma_{1}$ is bijective if and only if $196\left(a^{4}+2 a^{3} b+2 a^{2} b^{2}-2 a b^{3}+b^{4}\right)\left(a^{4}-\right.$ $\left.2 a^{3} b+2 a^{2} b^{2}+2 a b^{3}+b^{4}\right)\left(a^{24}+366 a^{20} b^{4}-705 a^{16} b^{8}+4772 a^{12} b^{12}-705 a^{8} b^{16}+\right.$ $\left.366 a^{4} b^{20}+b^{24}\right) \neq 0$.

(iii) By (7.7), (7.8), (7.10), we have

$$
\begin{aligned}
x^{10} y^{6} & =x^{9} y^{7}=x^{8} y^{8}=x^{7} y^{9}=x^{6} y^{10}=0, \quad x^{14} y^{2}=x^{2} y^{14}=0, \\
x^{16} & =-14 x^{12} y^{4}=14 x^{4} y^{12}=-y^{16}, \\
x^{15} y & =-14 x^{11} y^{5}=14 x^{3} y^{13}, \quad x y^{15}=-14 x^{5} y^{11}=14 x^{13} y^{3} .
\end{aligned}
$$


For $i=2$, by (7.14), the matrix representation of $\sigma_{2}$ with respect to the basis (7.0) is

$$
\left(\begin{array}{ccc}
-14 a b\left(14 a^{12}-143 a^{8} b^{4}+b^{12}\right) & -a^{2}\left(14 a^{12}-1001 a^{8} b^{4}+91 b^{12}\right) & 364 a^{3} b^{3}\left(a^{8}-b^{8}\right) \\
-364 a^{3} b^{3}\left(a^{8}-b^{8}\right) & -b^{2}\left(91 a^{12}-1001 a^{4} b^{8}+14 b^{12}\right) & -14 a b\left(a^{12}-143 a^{4} y^{8}+14 b^{12}\right) \\
a^{2}\left(14 a^{12}-1001 a^{8} b^{4}+91 b^{12}\right) & -364 a^{3} b^{3}\left(a^{8}-b^{8}\right) & -b^{2}\left(91 a^{12}-1001 a^{4} b^{8}+14 b^{12}\right)
\end{array}\right) .
$$

Therefore $\sigma_{2}$ is bijective if and only if $1372 a b(a-b)(a+b)\left(a^{2}+b^{2}\right)\left(a^{2}+2 a b-\right.$ $\left.b^{2}\right)\left(a^{2}-2 a b-b^{2}\right)\left(a^{4}+b^{4}\right)\left(a^{4}+6 a^{2} b^{2}+b^{4}\right)\left(2 a^{24}+3 a^{20} b^{4}+1506 a^{16} b^{8}+5170 a^{12} b^{12}+\right.$ $\left.1506 a^{8} b^{16}+3 a^{4} b^{20}+2 b^{24}\right) \neq 0$.

(iv) By (7.7), (7.8), (7.10), we have

$$
\begin{aligned}
& x^{9} y^{6}=x^{8} y^{7}=x^{7} y^{8}=x^{6} y^{9}=0, \\
& x^{15}=-14 x^{11} y^{4}=14 x^{3} y^{12}, \quad x^{14} y=-14 x^{10} y^{5}=14 x^{2} y^{13}, \\
& x y^{14}=-14 x^{5} y^{10}=14 x^{13} y^{2}, \quad y^{15}=-14 x^{4} y^{11}=14 x^{12} y^{3} .
\end{aligned}
$$

For $i=3$, by (7.15), the matrix representation of $\sigma_{3}$ with respect to the basis (7.0) is

$$
\left(\begin{array}{cccc}
-\left(14 a^{12}-495 a^{8} b^{4}+b^{12}\right) & 4 a b^{3}\left(55 a^{8}-3 b^{8}\right) & 66 a^{2} b^{2}\left(a^{8}-b^{8}\right) & 4 a^{3} b\left(3 a^{8}-55 b^{8}\right) \\
-24 a^{7} b\left(7 a^{4}-33 b^{4}\right) & -\left(14 a^{12}-495 a^{8} b^{4}+b^{12}\right) & 4 a b^{3}\left(55 a^{8}-3 b^{8}\right) & 66 a^{2} b^{2}\left(a^{8}-b^{8}\right) \\
-66 a^{2} b^{2}\left(a^{8}-b^{8}\right) & -4 a^{3} b\left(3 a^{8}-55 b^{8}\right) & -\left(a^{12}-495 a^{4} b^{8}+14 b^{12}\right) & 24 a b^{7}\left(33 a^{4}-7 b^{4}\right) \\
-4 a b^{3}\left(55 a^{8}-3 b^{8}\right) & -66 a^{2} b^{2}\left(a^{8}-b^{8}\right) & -4 a^{3} b\left(3 a^{8}-55 b^{8}\right) & -\left(a^{12}-495 a^{4} b^{8}+14 b^{12}\right)
\end{array}\right) .
$$

Therefore $\sigma_{3}$ is bijective if and only if $49\left(4 a^{48}-468 a^{44} b^{4}+39633 a^{40} b^{8}-\right.$ $590924 a^{36} b^{12}+3414840 a^{32} b^{16}+16202208 a^{28} b^{20}+28978278 a^{24} b^{24}+16202208 a^{20} b^{28}+$ $\left.3414840 a^{16} b^{32}-590924 a^{12} b^{36}+39633 a^{8} b^{40}-468 a^{4} b^{44}+4 b^{48}\right) \neq 0$.

(v) By (7.7), (7.8), (7.10), (7.11), we have

$$
\begin{aligned}
& x^{8} y^{6}=x^{7} y^{7}=x^{6} y^{8}=0, \quad x^{14}=-14 x^{10} y^{4}=14 x^{2} y^{12}, \\
& x^{13} y=-14 x^{9} y^{5}-x^{5} y^{9}, \quad x^{11} y^{3}=-x^{3} y^{11}, \\
& x y^{13}=-14 x^{5} y^{9}-x^{9} y^{5}, \quad y^{14}=-14 x^{4} y^{10}=14 x^{12} y^{2} .
\end{aligned}
$$

For $i=4$, by (7.16), the matrix representation of $\sigma_{4}$ with respect to the basis (7.0) is

$$
\left(\begin{array}{ccccc}
-14 a^{6}\left(a^{4}-15 b^{4}\right) & 120 a^{7} b^{3} & b^{2}\left(45 a^{8}-b^{8}\right) & 10 a b\left(a^{8}-b^{8}\right) & a^{2}\left(a^{8}-45 b^{8}\right) \\
-28 a^{5} b\left(5 a^{4}-9 b^{4}\right) & -14 a^{6}\left(a^{4}-15 b^{4}\right) & 120 a^{7} b^{3} & b^{2}\left(45 a^{8}-b^{8}\right) & 10 a b\left(a^{8}-b^{8}\right) \\
-10 a b\left(a^{8}-b^{8}\right) & -a^{2}\left(a^{8}-45 b^{8}\right) & 120 a^{3} b^{7} & 14 b^{6}\left(15 a^{4}-b^{4}\right) & 28 a b^{5}\left(9 a^{4}-5 b^{4}\right) \\
-b^{2}\left(45 a^{8}-b^{8}\right) & -10 a b\left(a^{8}-b^{8}\right) & -a^{2}\left(a^{8}-45 b^{8}\right) & 120 a^{3} b^{7} & 14 b^{6}\left(15 a^{4}-b^{4}\right) \\
-120 a^{7} b^{3} & -b^{2}\left(45 a^{8}-b^{8}\right) & -10 a b\left(a^{8}-b^{8}\right) & -a^{2}\left(a^{8}-45 b^{8}\right) & 120 a^{3} b^{7}
\end{array}\right) .
$$

Therefore $\sigma_{4}$ is bijective if and only if $-70 a b(a-b)(a+b)\left(a^{2}+b^{2}\right)\left(a^{2}+2 a b-\right.$ $\left.b^{2}\right)\left(a^{2}-2 a b-b^{2}\right)\left(a^{4}+b^{4}\right)\left(a^{4}+6 a^{2} b^{2}+b^{4}\right)\left(a^{4}+2 a^{3} b+2 a^{2} b^{2}-2 a b^{3}+b^{4}\right)\left(a^{4}-\right.$ $\left.2 a^{3} b+2 a^{2} b^{2}+2 a b^{3}+b^{4}\right)\left(2 a^{24}-637 a^{20} b^{4}+4066 a^{16} b^{8}+1330 a^{12} b^{12}+4066 a^{8} b^{16}-\right.$ $\left.637 a^{4} b^{20}+2 b^{24}\right) \neq 0$. 
(vi) By (7.7), (7.8), (7.11), we have

$$
\begin{aligned}
x^{7} y^{6} & =x^{6} y^{7}=0, \quad x^{13}=-14 x^{9} y^{4}-x^{5} y^{8} \\
x^{12} y & =-14 x^{8} y^{5}-x^{4} y^{9}, \quad x^{11} y^{2}=-x^{3} y^{10} \\
x^{2} y^{11} & =-x^{10} y^{3}, \quad x y^{12}=-14 x^{5} y^{8}-x^{9} y^{4}, \\
y^{13} & =-14 x^{4} y^{9}-x^{8} y^{5} .
\end{aligned}
$$

For $i=5$, by (7.17), the matrix representation of $\sigma_{5}$ with respect to the basis (7.0) is

$$
\left(\begin{array}{cccccc}
56 a^{5} b^{3} & 28 a^{6} b^{2} & 8 a^{7} b & a^{8}-b^{8} & -8 a b^{7} & -28 a^{2} b^{6} \\
-14\left(a^{4}-5 b^{4}\right) & 56 a^{5} b^{3} & 28 a^{6} b^{2} & 8 a^{7} b & a^{8}-b^{8} & -8 a b^{7} \\
-56 a^{3} b\left(2 a^{4}-b^{4}\right) & -14\left(a^{4}-5 b^{4}\right) & 56 a^{5} b^{3} & 28 a^{6} b^{2} & 8 a^{7} b & a^{8}-b^{8} \\
-\left(a^{8}-b^{8}\right) & 8 a b^{7} & 28 a^{2} b^{6} & 56 a^{3} b^{5} & 14 b^{4}\left(5 a^{4}-b^{4}\right) & 56 a b^{3}\left(a^{4}-2 b^{4}\right) \\
-8 a^{7} b & -\left(a^{8}-b^{8}\right) & 8 a b^{7} & 28 a^{2} b^{6} & 56 a^{3} b^{5} & 14 b^{4}\left(5 a^{4}-b^{4}\right) \\
-28 a^{6} b^{2} & -8 a^{7} b & -\left(a^{8}-b^{8}\right) & 8 a b^{7} & 28 a^{2} b^{6} & 56 a^{3} b^{5}
\end{array}\right) .
$$

Therefore $\sigma_{5}$ is bijective if and only if $a^{48}+4312 a^{44} b^{4}+348186 a^{40} b^{8}+392504 a^{36} b^{12}+$ $8766479 a^{32} b^{16}-37985808 a^{28} b^{20}+73725868 a^{24} b^{24}-37985808 a^{20} b^{28}+8766479 a^{16} b^{32}+$ $392504 a^{12} b^{36}+348186 a^{8} b^{40}+4312 a^{4} b^{44}+b^{48} \neq 0$.

(vii) By (7.11), we have

$$
\begin{aligned}
& x^{12}=-14 x^{8} y^{4}-x^{4} y^{8}, \quad x^{11} y=-14 x^{7} y^{5}-x^{3} y^{9}, \\
& x y^{11}=-14 x^{5} y^{7}-x^{9} y^{3}, \quad y^{12}=-14 x^{4} y^{8}-x^{8} y^{4} .
\end{aligned}
$$

For $i=6$, by (7.18), the matrix representation of $\sigma_{6}$ with respect to the basis (7.0) is

$$
\left(\begin{array}{ccccccc}
20 a^{3} b^{3} & 15 a^{4} b^{2} & 6 a^{5} b & a^{6} & 0 & -b^{6} & -6 a b^{5} \\
-a^{2}\left(14 a^{4}-15 b^{4}\right) & 20 a^{3} b^{3} & 15 a^{4} b^{2} & 6 a^{5} b & a^{6} & 0 & -b^{6} \\
-6 a b\left(14 a^{4}-b^{4}\right) & -a^{2}\left(14 a^{4}-15 b^{4}\right) & 20 a^{3} b^{3} & 15 a^{4} b^{2} & 6 a^{5} b & a^{6} & 0 \\
0 & b^{6} & 6 a b^{5} & 15 a^{2} b^{4} & 20 a^{3} b^{3} & b^{2}\left(15 a^{4}-14 b^{4}\right) & 6 a b\left(a^{4}-14 b^{4}\right) \\
-a^{6} & 0 & b^{6} & 6 a b^{5} & 15 a^{2} b^{4} & 20 a^{3} b^{3} & b^{2}\left(15 a^{4}-14 b^{4}\right) \\
-6 a^{5} b & -a^{6} & 0 & b^{6} & 6 a b^{5} & 15 a^{2} b^{4} & 20 a^{3} b^{3} \\
-15 a^{4} b^{2} & -6 a^{5} b & -a^{6} & 0 & b^{6} & 6 a b^{5} & 15 a^{2} b^{4}
\end{array}\right) .
$$

Therefore $\sigma_{6}$ is bijective if and only if $-6 a b(a-b)(a+b)\left(a^{2}+b^{2}\right)\left(a^{2}+2 a b-\right.$ $\left.b^{2}\right)\left(a^{2}-2 a b-b^{2}\right)\left(a^{4}+b^{4}\right)\left(a^{4}+6 a^{2} b^{2}+b^{4}\right)\left(a^{24}-470 a^{20} b^{4}+2639 a^{16} b^{8}-244 a^{12} b^{12}+\right.$ $\left.2639 a^{8} b^{16}-470 a^{4} b^{20}+b^{24}\right) \neq 0$.

(viii) By (7.11), we have

$$
\begin{aligned}
& x^{11}=-14 x^{7} y^{4}-x^{3} y^{8}, \quad x^{10} y=-14 x^{6} y^{5}-x^{2} y^{9}, \\
& x y^{10}=-14 x^{5} y^{6}-x^{9} y^{2}, \quad y^{11}=-14 x^{4} y^{7}-x^{8} y^{3} .
\end{aligned}
$$


For $i=7$, by (7.19), the matrix representation of $\sigma_{7}$ with respect to the basis (7.0) is

$$
\left(\begin{array}{cccccccc}
6 a^{2} b^{2} & 4 a^{3} b & a^{4} & 0 & 0 & 0 & -b^{4} & -4 a b^{3} \\
4 a b^{3} & 6 a^{2} b^{2} & 4 a^{3} b & a^{4} & 0 & 0 & 0 & -b^{4} \\
-\left(14 a^{4}-b^{4}\right) & 4 a b^{3} & 6 a^{2} b^{2} & 4 a^{3} b & a^{4} & 0 & 0 & 0 \\
-56 a^{3} b & -\left(14 a^{4}-b^{4}\right) & 4 a b^{3} & 6 a^{2} b^{2} & 4 a^{3} b & a^{4} & 0 & 0 \\
0 & 0 & b^{4} & 4 a b^{3} & 6 a^{2} b^{2} & 4 a^{3} b & a^{4}-14 b^{4} & -56 a b^{3} \\
0 & 0 & 0 & b^{4} & 4 a b^{3} & 6 a^{2} b^{2} & 4 a^{3} b & a^{4}-14 b^{4} \\
-a^{4} & 0 & 0 & 0 & b^{4} & 4 a b^{3} & 6 a^{2} b^{2} & 4 a^{3} b \\
-4 a^{3} b & -a^{4} & 0 & 0 & 0 & b^{4} & 4 a b^{3} & 6 a^{2} b^{2}
\end{array}\right) .
$$

Therefore $\sigma_{7}$ is bijective if and only if $\left(a^{4}+2 a^{3} b+2 a^{2} b^{2}-2 a b^{3}+b^{4}\right)^{4}\left(a^{4}-2 a^{3} b+\right.$ $\left.2 a^{2} b^{2}+2 a b^{3}+b^{4}\right)^{4} \neq 0$.

(ix) By (7.11), we have

$$
\begin{aligned}
& x^{10}=-14 x^{6} y^{4}-x^{2} y^{8}, \quad x^{9} y=-14 x^{5} y^{5}-x y^{9}, \\
& y^{10}=-14 x^{4} y^{6}-x^{8} y^{2} .
\end{aligned}
$$

For $i=8$, by (7.20), the matrix representation of $\sigma_{8}$ with respect to the basis (7.0) is

$$
\left(\begin{array}{cccccccc}
2 a b & a^{2} & 0 & 0 & 0 & 0 & 0 & -b^{2} \\
b^{2} & 2 a b & a^{2} & 0 & 0 & 0 & 0 & 0 \\
0 & b^{2} & 2 a b & a^{2} & 0 & 0 & 0 & 0 \\
-14 a^{2} & 0 & b^{2} & 2 a b & a^{2} & 0 & 0 & 0 \\
0 & 0 & 0 & b^{2} & 2 a b & a^{2} & 0 & -14 b^{2} \\
0 & 0 & 0 & 0 & b^{2} & 2 a b & a^{2} & 0 \\
0 & 0 & 0 & 0 & 0 & b^{2} & 2 a b & a^{2} \\
-a^{2} & 0 & 0 & 0 & 0 & 0 & b^{2} & 2 a b
\end{array}\right) .
$$

Therefore $\sigma_{8}$ is bijective if and only if $\left(a^{4}+2 a^{3} b+2 a^{2} b^{2}-2 a b^{3}+b^{4}\right)^{2}\left(a^{4}-2 a^{3} b+\right.$ $\left.2 a^{2} b^{2}+2 a b^{3}+b^{4}\right)^{2} \neq 0$.

(x) For $i=9, \sigma_{9}$ is bijective obviously.

By (i) $\sim(x)$, we get (1).

(2) For $1 \leq i \leq 11$, we get the same polynomials $g_{i}$ as in Theorem 6.1(1). Thus we have the same $B\left(g_{i}\right)(1 \leq i \leq 11)$ as in Theorem 6.1 (2).

\section{The Lefschetz elements for $G_{22}$}

We recall that the group $G_{22}$ of order 240 contains a binary icosahedral group of order 120 as a subgroup of index 2 . In this section we give a characterization of the strong Lefschetz element of the coinvariant algebras of $G_{22}$. Let $G=G_{22}$ :

$$
G=\left\langle\frac{1}{\sqrt{5}} i\left(\begin{array}{cc}
\eta^{4}-\eta & \eta^{2}-\eta^{3} \\
\eta^{2}-\eta^{3} & \eta-\eta^{4}
\end{array}\right), \frac{1}{\sqrt{5}}\left(\begin{array}{cc}
\eta^{2}-\eta^{4} & \eta^{4}-1 \\
1-\eta & \eta^{3}-\eta
\end{array}\right)\right\rangle, \quad\left(\eta=e^{\frac{2 \pi i}{5}}\right) .
$$

The invariant algebra of $G$ is

$$
S(V)^{G}=\mathbf{C}\left[X^{11} Y+11 X^{6} Y^{6}-X Y^{11},-\left(X^{20}+Y^{20}\right)+228\left(X^{15} Y^{5}-X^{5} Y^{15}\right)-494 X^{10} Y^{10}\right] .
$$


By (3) in 3.2 and Table 1, we have

$$
\begin{aligned}
P_{S(V)_{G}}(t)= & \frac{\left(1-t^{12}\right)\left(1-t^{20}\right)}{(1-t)^{2}} \\
= & 1+2 t+3 t^{2}+4 t^{3}+5 t^{4}+6 t^{5}+7 t^{6}+8 t^{7}+9 t^{8}+10 t^{9}+11 t^{10}+12 t^{11} \\
& +\cdots+12 t^{19}+11 t^{20}+10 t^{21}+9 t^{22}+8 t^{23}+7 t^{24}+6 t^{25}+5 t^{26}+4 t^{27} \\
& +3 t^{28}+2 t^{29}+t^{30}
\end{aligned}
$$

We choose a basis of $S(V)_{G}$

$$
\begin{aligned}
& \left\{1, x, y, x^{2}, x y, y^{2}, x^{3}, x^{2} y, x y^{2}, y^{3}, x^{4}, x^{3} y, x^{2} y^{2}, x y^{3}, y^{4}, x^{5}, x^{4} y, x^{3} y^{2}, x^{2} y^{3}, x y^{4}, y^{5},\right. \\
& x^{6}, x^{5} y, x^{4} y^{2}, x^{3} y^{3}, x^{2} y^{4}, x y^{5}, y^{6}, x^{7}, x^{6} y, x^{5} y^{2}, x^{4} y^{3}, x^{3} y^{4}, x^{2} y^{5}, x y^{6}, y^{7}, x^{8}, x^{7} y, x^{6} y^{2}, \\
& x^{5} y^{3}, x^{4} y^{4}, x^{3} y^{5}, x^{2} y^{6}, x y^{7}, y^{8}, x^{9}, x^{8} y, x^{7} y^{2}, x^{6} y^{3}, x^{5} y^{4}, x^{4} y^{5}, x^{3} y^{6}, x^{2} y^{7}, x y^{8}, y^{9}, x^{10}, \\
& x^{9} y, x^{8} y^{2}, x^{7} y^{3}, x^{6} y^{4}, x^{5} y^{5}, x^{4} y^{6}, x^{3} y^{7}, x^{2} y^{8}, x y^{9}, y^{10}, x^{11}, x^{10} y, x^{9} y^{2}, x^{8} y^{3}, x^{7} y^{4}, x^{6} y^{5} \text {, } \\
& x^{5} y^{6}, x^{4} y^{7}, x^{3} y^{8}, x^{2} y^{9}, x y^{10}, y^{11}, x^{12}, x^{11} y, x^{10} y^{2}, x^{9} y^{3}, x^{8} y^{4}, x^{7} y^{5}, x^{5} y^{7}, x^{4} y^{8}, x^{3} y^{9}, \\
& x^{2} y^{10}, x y^{11}, y^{12}, x^{13}, x^{12} y, x^{11} y^{2}, x^{10} y^{3}, x^{9} y^{4}, x^{8} y^{5}, x^{5} y^{8}, x^{4} y^{9}, x^{3} y^{10}, x^{2} y^{11}, x y^{12}, y^{13} \text {, } \\
& x^{14}, x^{13} y, x^{12} y^{2}, x^{11} y^{3}, x^{10} y^{4}, x^{9} y^{5}, x^{5} y^{9}, x^{4} y^{10}, x^{3} y^{11}, x^{2} y^{12}, x y^{13}, y^{14}, x^{15}, x^{14} y, x^{13} y^{2} \text {, } \\
& x^{12} y^{3}, x^{11} y^{4}, x^{10} y^{5}, x^{5} y^{10}, x^{4} y^{11}, x^{3} y^{12}, x^{2} y^{13}, x y^{14}, y^{15}, x^{16}, x^{15} y, x^{14} y^{2}, x^{13} y^{3}, x^{12} y^{4} \text {, } \\
& x^{11} y^{5}, x^{5} y^{11}, x^{4} y^{12}, x^{3} y^{13}, x^{2} y^{14}, x y^{15}, y^{16}, x^{17}, x^{16} y, x^{15} y^{2}, x^{14} y^{3}, x^{13} y^{4}, x^{12} y^{5}, x^{5} y^{12} \text {, } \\
& x^{4} y^{13}, x^{3} y^{14}, x^{2} y^{15}, x y^{16}, y^{17}, x^{18}, x^{17} y, x^{16} y^{2}, x^{15} y^{3}, x^{14} y^{4}, x^{13} y^{5}, x^{5} y^{13}, x^{4} y^{14}, x^{3} y^{15} \text {, } \\
& x^{2} y^{16}, x y^{17}, y^{18}, x^{19}, x^{18} y, x^{17} y^{2}, x^{16} y^{3}, x^{15} y^{4}, x^{14} y^{5}, x^{5} y^{14}, x^{4} y^{15}, x^{3} y^{16}, x^{2} y^{17}, x y^{18} \text {, } \\
& y^{19}, x^{19} y, x^{18} y^{2}, x^{17} y^{3}, x^{16} y^{4}, x^{15} y^{5}, x^{5} y^{15}, x^{4} y^{16}, x^{3} y^{17}, x^{2} y^{18}, x y^{19}, y^{20}, x^{20} y, x^{19} y^{2} \text {, } \\
& x^{18} y^{3}, x^{17} y^{4}, x^{16} y^{5}, x^{5} y^{16}, x^{4} y^{17}, x^{3} y^{18}, x^{2} y^{19}, x y^{20}, x^{20} y^{2}, x^{19} y^{3}, x^{18} y^{4}, x^{17} y^{5}, x^{5} y^{17} \text {, } \\
& x^{4} y^{18}, x^{3} y^{19}, x^{2} y^{20}, x y^{21}, x^{21} y^{2}, x^{20} y^{3}, x^{19} y^{4}, x^{18} y^{5}, x^{5} y^{18}, x^{4} y^{19}, x^{3} y^{20}, x^{2} y^{21}, x^{21} y^{3} \text {, } \\
& x^{20} y^{4}, x^{19} y^{5}, x^{5} y^{19}, x^{4} y^{20}, x^{3} y^{21}, x^{2} y^{22}, x^{22} y^{3}, x^{21} y^{4}, x^{20} y^{5}, x^{5} y^{20}, x^{4} y^{21}, x^{3} y^{22}, x^{22} y^{4} \text {, } \\
& x^{21} y^{5}, x^{5} y^{21}, x^{4} y^{22}, x^{3} y^{23}, x^{23} y^{4}, x^{22} y^{5}, x^{5} y^{22}, x^{4} y^{23}, x^{23} y^{5}, x^{5} y^{23}, x^{4} y^{24}, x^{24} y^{5}, x^{5} y^{24} \text {, }
\end{aligned}
$$

THEOREM 7. (1) The element $l=a x+b y \in S_{1}(V)_{G}$ is the strong Lefschetz element if and only if $\prod_{i=1}^{21} g_{i} \neq 0$, where $g_{i}$ is a polynomial in $a, b$ given by

$g_{1}(a, b)=a$,

$g_{2}(a, b)=b$,

$g_{3}(a, b)=a^{2}+a b-b^{2}$,

$g_{4}(a, b)=a^{2}+b^{2}$,

$g_{5}(a, b)=a^{4}-3 a^{3} b+4 a^{2} b^{2}-2 a b^{3}+b^{4}$, 
$g_{6}(a, b)=a^{4}+2 a^{3} b+4 a^{2} b^{2}+3 a b^{3}+b^{4}$,

$g_{7}(a, b)=a^{4}-3 a^{3} b-a^{2} b^{2}+3 a b^{3}+b^{4}$,

$g_{8}(a, b)=a^{4}+2 a^{3} b-6 a^{2} b^{2}-2 a b^{3}+b^{4}$,

$g_{9}(a, b)=a^{8}+4 a^{7} b+7 a^{6} b^{2}+2 a^{5} b^{3}+15 a^{4} b^{4}-2 a^{3} b^{5}+7 a^{2} b^{6}-4 a b^{7}+b^{8}$,

$g_{10}(a, b)=a^{8}-a^{7} b+7 a^{6} b^{2}+7 a^{5} b^{3}-7 a^{3} b^{5}+7 a^{2} b^{6}+a b^{7}+b^{8}$,

$g_{11}(a, b)=a^{8}-a^{6} b^{2}+a^{4} b^{4}-a^{2} b^{6}+b^{8}$,

$g_{12}(a, b)=a^{8}+4 a^{7} b+17 a^{6} b^{2}+22 a^{5} b^{3}+5 a^{4} b^{4}-22 a^{3} b^{5}+17 a^{2} b^{6}-4 a b^{7}+b^{8}$,

$g_{13}(a, b)=a^{8}-6 a^{7} b+17 a^{6} b^{2}-18 a^{5} b^{3}+25 a^{4} b^{4}+18 a^{3} b^{5}+17 a^{2} b^{6}+6 a b^{7}+b^{8}$,

$g_{14}(a, b)=56 a^{60}-93411 a^{55} b^{5}+5785419 a^{50} b^{10}-767941695 a^{45} b^{15}$

$+3616437090 a^{40} b^{20}-11153212659 a^{35} b^{25}-9286142171 a^{30} b^{30}+111532126594 a^{25} b^{35}$

$+3616437090 a^{20} b^{40}+767941695 a^{15} b^{45}+5785419 a^{10} b^{50}+93411 a^{5} b^{55}+56 b^{60}$,

$g_{15}(a, b)=21 a^{60}+124424 a^{55} b^{5}+10939454 a^{50} b^{10}-95837120 a^{45} b^{15}$

$+3443405315 a^{40} b^{20}+6913090956 a^{35} b^{25}+18005759264 a^{30} b^{30}-6913090956 a^{25} b^{35}$

$+3443405315 a^{20} b^{40}+95837120 a^{15} b^{45}+10939454 a^{10} b^{50}-124424 a^{5} b^{55}+21 b^{60}$,

$g_{16}(a, b)=781 a^{60}-168636 a^{55} b^{5}+143062194 a^{50} b^{10}-9343436820 a^{45} b^{15}$

$+65281931715 a^{40} b^{20}-76630172184 a^{35} b^{25}+23325796604 a^{30} b^{30}+76630172184 a^{25} b^{35}$

$+65281931715 a^{20} b^{40}+9343436820 a^{15} b^{45}+143062194 a^{10} b^{50}+168636 a^{5} b^{55}+781 b^{60}$,

$g_{17}(a, b)=781 a^{60}-2856636 a^{55} b^{5}-4777806 a^{50} b^{10}-12582476820 a^{45} b^{15}$

$+30096011715 a^{40} b^{20}-263674652184 a^{35} b^{25}-338911771396 a^{30} b^{30}+263674652184 a^{25} b^{35}$

$+30096011715 a^{20} b^{40}+12582476820 a^{15} b^{45}-4777806 a^{10} b^{50}+2856636 a^{5} b^{55}+781 b^{60}$,

$g_{18}(a, b)=781 a^{60}+9833796 a^{55} b^{5}+693195954 a^{50} b^{10}+2709493740 a^{45} b^{15}$

$+196213766595 a^{40} b^{20}+619389058536 a^{35} b^{25}+1371263535356 a^{30} b^{30}-619389058536 a^{25} b^{35}$

$+196213766595 a^{20} b^{40}-2709493740 a^{15} b^{45}+693195954 a^{10} b^{50}-9833796 a^{5} b^{55}+781 b^{60}$,

$g_{19}(a, b)=a^{60}-184 a^{55} b^{5}+184934 a^{50} b^{10}-11924960 a^{45} b^{15}$

$+84005495 a^{40} b^{20}-95896644 a^{35} b^{25}+34168576 a^{30} b^{30}+95896644 a^{25} b^{35}$

$+84005495 a^{20} b^{40}+11924960 a^{15} b^{45}+184934 a^{10} b^{50}+184 a^{5} b^{55}+b^{60}$,

$g_{20}(a, b)=781 a^{120}+1056728 a^{115} b^{5}+1690618404 a^{110} b^{10}$

$+335172561432 a^{105} b^{15}+36048914871226 a^{100} b^{20}-3695470971608808 a^{95} b^{25}$

$+130053725826919236 a^{90} b^{30}-1594868948748657512 a^{85} b^{35}+8098914234602656899 a^{80} b^{40}$

$-5824418933856860288 a^{75} b^{45}+30737529948917758104 a^{70} b^{50}+11758899072173963712 a^{65} b^{55}$

$+38124609592305613676 a^{60} b^{60}-11758899072173963712 a^{55} b^{65}+30737529948917758104 a^{50} b^{70}$

$+5824418933856860288 a^{45} b^{75}+8098914234602656899 a^{40} b^{80}+1594868948748657512 a^{35} b^{85}$ 


$$
\begin{aligned}
& +130053725862919236 a^{30} b^{90}+3695470971608808 a^{25} b^{95}+36048914871226 a^{20} b^{100} \\
& -335172561432 a^{15} b^{105}+1690618404 a^{10} b^{100}-1056728 a^{5} b^{115}+781 b^{120} \\
& g_{21}(a, b)=781 a^{120}-37434408 a^{115} b^{5}+28669521444 a^{110} b^{10} \\
& -4018508591592 a^{105} b^{15}+231220616958906 a^{100} b^{20}+13323015986481432 a^{95} b^{25} \\
& +4830395349952 a^{90} b^{30}+1093401918957423768 a^{85} b^{35}+8911688602181278851 a^{80} b^{40} \\
& -74863050767566013568 a^{75} b^{45}-21436724021848920936 a^{70} b^{50}+969892095324814208448 a^{65} b^{55} \\
& -544495324964301526164 a^{60} b^{60}-969892095324814208448 a^{55} b^{65}-21436724021848920936 a^{50} b^{70} \\
& +74863050767566013568 a^{45} b^{75}+8911688602181278851 a^{40} b^{80}-1093401918957423768 a^{35} b^{85} \\
& +483039534995219524 a^{30} b^{90}-13323015986481432 a^{25} b^{95}+231220616958906 a^{20} b^{100} \\
& +4018508591592 a^{15} b^{105}+28669521444 a^{10} b^{110}+37434408 a^{5} b^{115}+781 b^{120}
\end{aligned}
$$

(See the end of $\$ 3$ for the notation.)

(2) We take an icosahedron in $R^{3}$. Put Z-axis on a diameter of $S^{2}$ passing through 2 vertices of the icosahedron and $Y$-axis on a diameter of $S^{2}$ passing through 2 centers of edges of the icosahedron which is perpendicular to Z-axis. Then the sets $B\left(g_{i}\right)$, which are the inverse images of $t\left(g_{i}\right)(1 \leq i \leq 13)$ under the stereographic projection, are given as follows (see the end of $\$ 3$ for the notation) :

(i) $B\left(g_{1}\right), B\left(g_{2}\right), B\left(g_{3}\right), B\left(g_{5}\right), B\left(g_{6}\right)$ are the twelve vertices of the icosahedron.

(ii) $B\left(g_{4}\right), B\left(g_{8}\right), B\left(g_{11}\right), B\left(g_{12}\right), B\left(g_{13}\right)$ are the thirty points of $S^{2}$ corresponding to the centers of the edges of the icosahedron.

(iii) $B\left(g_{7}\right), B\left(g_{9}\right), B\left(g_{10}\right)$ are the twenty points of $S^{2}$ corresponding to the centers of the faces of the icosahedron.

Proof. (1) Since $I_{G}=\left(X^{11} Y+11 X^{6} Y^{6}-X Y^{11},-\left(X^{20}+Y^{20}\right)+228\left(X^{15} Y^{5}-\right.\right.$ $\left.\left.X^{5} Y^{15}\right)-494 X^{10} Y^{10}\right)$, we have

$$
\begin{gathered}
x^{11} y+11 x^{6} y^{6}-x y^{11}=0, \\
-\left(x^{20}+y^{20}\right)+228\left(x^{15} y^{5}-x^{5} y^{15}\right)-494 x^{10} y^{10}=0 .
\end{gathered}
$$

For later use, we prepare several relations. By (8.1), we have

$$
x^{6} y^{6}=-\frac{1}{11}\left(x^{11} y-x y^{11}\right) \text {. }
$$

By (8.2), (8.3), we have

$$
\begin{aligned}
x^{20} & =-y^{20}+228\left(x^{15} y^{5}-x^{5} y^{15}\right)+494 x^{4} y^{4} \cdot \frac{1}{11}\left(x^{11} y-x y^{11}\right) \\
& =-y^{20}+\frac{3002}{11}\left(x^{15} y^{5}-x^{5} y^{15}\right) .
\end{aligned}
$$


By (8.3), (8.4), we have

$$
\begin{aligned}
x^{11} y^{11} & =-\frac{1}{11} x y\left(x^{15} y^{5}-x^{5} y^{15}\right) \\
& =-\frac{1}{11} x y \cdot \frac{11}{3002}\left(x^{20}+y^{20}\right) \\
& =-\frac{1}{3002}\left(x^{21} y+x y^{21}\right) .
\end{aligned}
$$

Moreover since

$$
\begin{aligned}
x^{11} y^{11} & =-\frac{1}{11}\left(x^{16} y^{6}-x^{6} y^{16}\right) \\
& =-\frac{1}{11}\left(-x^{10} \cdot \frac{1}{11}\left(x^{11} y-x y^{11}\right)+y^{10} \cdot \frac{1}{11}\left(x^{11} y-x y^{11}\right)\right) \\
& =\frac{1}{121}\left(x^{21} y+x y^{21}-2 x^{11} y^{11}\right),
\end{aligned}
$$

we have

$$
x^{11} y^{11}=\frac{1}{123}\left(x^{21} y+x y^{21}\right) .
$$

Therefore by (8.5), (8.6), we have

$$
x^{11} y^{11}=x^{21} y+x y^{21}=0 .
$$

By (8.3), (8.7), we have

$$
\begin{aligned}
x^{16} y^{6} & =-\frac{1}{11}\left(x^{21} y-x^{11} y^{11}\right) \\
& =-\frac{1}{11} x^{21} y \\
& =\frac{1}{11} x y^{21}, \\
x^{6} y^{16} & =-\frac{1}{11}\left(x^{11} y^{11}-x y^{21}\right) \\
& =x y^{21} .
\end{aligned}
$$

Therefore we have

$$
x^{16} y^{6}=x^{6} y^{16}=-\frac{1}{11} x^{21} y=\frac{1}{11} x y^{21} .
$$

Multiplying (8.8) by $x^{5}$ and using (8.7), we have

$$
x^{26} y=x^{21} y^{6}=x^{6} y^{21}=x y^{26}=0 .
$$


Therefore by (8.7), (8.9), we have

$$
x^{i} y^{j}=x^{j} y^{i}=0, \quad i, j \geq 11
$$

and

$$
x^{i} y^{j}=x^{j} y^{i}=0, \quad i \geq 26, \quad j \geq 1 \quad \text { or } \quad i \geq 21, \quad j \geq 6 .
$$

Since $x^{21} y=-x y^{21}$, we have

$$
x^{i+21} y^{j+1}=-x^{i+1} y^{j+21} .
$$

By (8.8), we have

$$
\begin{aligned}
& x^{i} y^{j}=x^{i+10} y^{j-10}=-\frac{1}{11} x^{i+15} y^{j-15}=\frac{1}{11} x^{i-5} y^{j+5}, \quad i \geq 6, j \geq 16, \\
& x^{i} y^{j}=x^{i-10} y^{j+10}=-\frac{1}{11} x^{i+5} y^{j-5}=\frac{1}{11} x^{i-15} y^{j+15}, \quad i \geq 16, j \geq 6 .
\end{aligned}
$$

It follows from (8.4), (8.10), (8.13), (8.14) that

$$
x^{30-i}=273 x^{25-i} y^{5}, \quad y^{30-i}=-273 x^{5} y^{25-i} .
$$

By (8.3), we have

$$
\begin{aligned}
& x^{15-i} y^{10-j}=\frac{1}{122}\left(-11 x^{20-i} y^{5-j}+x^{5-i} y^{20-j}\right), \quad 0 \leq i, j \leq 4, \\
& x^{10-i} y^{15-j}=\frac{1}{122}\left(x^{20-i} y^{5-j}+11 x^{5-i} y^{20-j}\right), \quad 0 \leq i, j \leq 4 .
\end{aligned}
$$

It follows from (8.4), (8.16), (8.17) that

$$
\begin{array}{ll}
x^{25-i}=\frac{11}{61}\left(-142 x^{5-i} y^{20}+1501 x^{20-i} y^{5}\right), & 0 \leq i, j \leq 4, \\
y^{25-i}=-\frac{11}{61}\left(142 x^{20} y^{5-i}+1501 x^{5} y^{20-i}\right), & 0 \leq i, j \leq 4 .
\end{array}
$$

By (8.3), we have

$$
x^{10-i} y^{10-j}=-\frac{1}{11}\left(x^{15-i} y^{5-j}-x^{5-i} y^{15-j}\right), \quad 0 \leq i, j \leq 4,
$$

By using (8.10), ., (8.20), we get the matrix representations of $\rho_{i}, i=0, \ldots, 15$. But we omit the details for brevity's sake.

(2) For $1 \leq i \leq 21$, we have the following:

$$
\begin{aligned}
g_{1} g_{2} g_{3} g_{5} g_{6} & =a^{11} b+11 a^{6} b^{6}-a b^{11}, \\
g_{4} g_{8} g_{11} g_{12} g_{13} & =-\left(a^{20}+b^{20}\right)+228\left(a^{15} b^{5}-a^{5} b^{15}\right)-494 a^{10} b^{10}, \\
g_{7} g_{9} g_{10} & =\left(a^{30}+b^{30}\right)+522\left(a^{25} b^{5}-a^{5} b^{25}\right)-10005\left(a^{20} b^{10}+a^{10} b^{20}\right) .
\end{aligned}
$$


These polynomials are generators of the invariant algebra of the binary icosahedral group ([5], chap. 2). The sets of zeros of these invariants correspond to the set of vertices, the centers of the edges and the centers of the faces of the icosahedron respectively.

\section{References}

[ 1 ] Y. Gomi, I. NAKAmura and K. Shinoda, Coinvariant algebras of finite subgroups of $S L(3$, C), Cana. J. Math. 56 (4) (2004), 495-528.

[ 2 ] T. Harima, J. C. Migliore, U. NAgel and J. Watanabe, The weak and strong Lefschetz properties for Artinian k-algebras, J. Algebra 262 (2003), 99-126.

[3] T. HARIMA and A. WACHI, Generic initial ideals, graded Betti numbers and $k$-Lefschetz properties, arXiv:0707.2247 (2007).

[ 4 ] H. Hiller, Geometry of Coxeter Groups, Pitman Publishing, Boston, 1982.

[ 5 ] F. KLEIN, Vorlesungen uber das Ikosaeder und die Auflosung der Gleichungen vom funften Grade [Lectures on the icosahedron and the solution of equations of the fifth degree], Reprint of the 1884 original, ed. P. SLODOWY, Birkhauser (1993).

[ 6 ] T. Maeno, Y. Numata and A. WACHI, Strong Lefschetz elements of the coinvariant rings of finite Coxeter groups, preprint, math.RT/0809.3558 (2008).

[ 7 ] T. MAEno and J. Watanabe, Lefschetz elements of Artinian Gorenstein algebras and Hessians of homogeneous polynomials, arXiv:0903.3581 (2009).

[ 8 ] J. Matsuzawa, Singularities and Root systems, Asakurashoten, Tokyo (2002), (in Japanese).

[9] C. R. MCDANIEL, The strong Lefschetz property for coinvariant rings of finite reflection groups, arXiv:0909.4184 (2009).

[10] Y. NUmATA and A. WACHI, The strong Lefschetz property of the coinvariant ring of the Coxeter group of type $H_{4}$, J. Algebra 318 (2007), 1032-1038.

[11] G. C. Shephard and J. A. Todd, Finite unitary reflection groups, Cana. J. Math. 6 (1954), $274-304$.

[12] T. A. Springer, Invariant Theory, Lecture Notes in Math., no. 585, Springer Verlag, Berlin (1977).

[13] R. P. Stanley, Weyl groups, the hard Lefschetz theorem and the Sperner property, Siam. J. Alg. and Disc. Meth. 1 (1980), 168-184.

[14] R. P. Stanley, Combinatorial Applications of the Hard Lefschetz Theorem, In Proceedings International Congress of Mathematicians (Warsaw, 1983): 447-453.

Present Address:

SACHIKO YoshidA 